\title{
Geometry of Hermitian manifolds
}

\author{
Kefeng Liu, Xiaokui Yang
}

\begin{abstract}
On Hermitian manifolds, the second Ricci curvature tensors of various metric connections are closely related to the geometry of Hermitian manifolds. By refining the Bochner formulas for any Hermitian complex vector bundle (Riemannain real vector bundle) with an arbitrary metric connection over a compact Hermitian manifold, we can derive various vanishing theorems for Hermitian manifolds and complex vector bundles by the second Ricci curvature tensors. We will also introduce a natural geometric flow on Hermitian manifolds by using the second Ricci curvature tensor.
\end{abstract}

\section{Introduction}

It is well-known([5]) that on a compact Kähler manifold, if the Ricci curvature is positive, then the first Betti number is zero; if the Ricci curvature is negative, then there is no holomorphic vector field. The key ingredient for the proofs of such results is the Kähler symmetry. On the other hand, on an Hermitian manifold, we don't have such symmetry and there are several different Ricci curvatures. While on a Kähler manifold, all these Ricci curvatures coincide, since the Chern curvature on a Kähler manifold coincides with the curvature of the (complexified) Levi-Civita connection. We can see this more clearly on an abstract Hermitian holomorphic bundle $E$. The Chern connection $\nabla^{C H}$ on $E$ is the unique connection which is compatible with the holomorphic structure and the Hermitian metric on $E$. Hence, the Chern curvature $\Theta^{E} \in \Gamma\left(M, \Lambda^{1,1} T^{*} M \otimes E^{*} \otimes E\right)$. There are two methods to take trace of $\Theta^{E}$. If we take trace of $\Theta^{E}$ on the part $E n d(E)=E^{*} \otimes E$, we get a $(1,1)$-form on $M$ which it is called the first Ricci curvature. It is well known that the first Ricci curvature represents the first Chern class of the bundle. On the other hand, if we take trace on the $(1,1)$-part using the metric of the manifold, we obtain an endomorphism of $E, \operatorname{Tr}_{\omega} \Theta^{E} \in \Gamma\left(M, E^{*} \otimes E\right)$. It is called the second Ricci curvature of $\Theta^{E}$. The first and second Ricci curvatures have different geometric meanings, which were not clearly studied in some earlier literatures. We should point out that the nonexistence of holomorphic sections is characterized by the second Ricci curvature. Let $E$ be the holomorphic tangent bundle $T^{1,0} M$. If $M$ is Kähler, the first and second Ricci curvature are the same by the Kähler symmetry. Unfortunately, on an Hermitian manifold, the Chern curvature is not symmetric, i.e., the first and second Ricci curvatures are different. Moreover, in general they can not be compared. An interesting example is the Hopf manifold $\mathbb{S}^{2 n+1} \times \mathbb{S}^{1}$. The canonical metric on it has strictly positive second Ricci curvature!

In this paper, we study the nonexistence of holomorphic and harmonic sections of an abstract vector bundle over a compact Hermitian manifold. Let $E$ be a holomorphic vector bundle over a compact Hermitian manifold $(M, \omega)$. Since the holomorphic section space $H^{0}(M, E)$ is independent on the connections of $E$, we can choose any connection on it. As we mentioned above, the key part, is the second Ricci curvature of the connection. For example, on the holomorphic tangent bundle $T^{1,0} M$ of an Hermitian manifold $M$, there are three common connections 
(1) the complexified Levi-Civita connection $\nabla$ on $T^{1,0} M$;

(2) the Chern connection $\nabla^{C H}$ on $T^{1,0} M$;

(3) the Bismut connection $\nabla^{B}$ on $T^{1,0} M$.

It is well-known that if $M$ is Kähler, all three connections are the same. However, in general, the relations among them are somewhat mysterious. In this paper, we derive certain relations about their curvatures on certain Hermitian manifolds.

Let $E$ be an Hermitian complex (possibly non-holomorphic) vector bundle or a Riemannian real vector bundle over a compact Hermitian manifold $(M, \omega)$. Let $\partial_{E}, \bar{\partial}_{E}$ be the $(1,0),(0,1)$ part of $\nabla^{E}$ respectively. The $(1,1)$-curvature of $\nabla^{E}$ is denoted by $R^{E} \in \Gamma\left(M, \Lambda^{1,1} T^{*} M \otimes\right.$ $\left.E^{*} \otimes E\right)$. It can be viewed as a representation of the operator $\partial_{E} \bar{\partial}_{E}+\bar{\partial}_{E} \partial_{E}$. We can define harmonic section spaces associated to $\left(E, \nabla^{E}\right)$ by

$$
\mathcal{H}_{\bar{\partial}_{E}}^{p, q}(M, E)=\left\{\varphi \in \Omega^{p, q}(M, E) \mid \bar{\partial}_{E} \varphi=\bar{\partial}_{E}^{*} \varphi=0\right\}
$$

In general, on a complex vector bundle $E$, there is no such terminology like "holomorphic section of $E$ ". However, if the vector bundle $E$ is holomorphic and $\nabla^{E}$ is the Chern connection on $E$ i.e. $\bar{\partial}_{E}=\bar{\partial}$, then $\mathcal{H}_{\bar{\partial}_{E}, q}^{p}(M, E)$ is isomorphic to the Dolbeault cohomology group $H_{\bar{\partial}}^{p, q}(M, E)$ and $H_{\bar{\partial}}^{0}(M, E)$ is the holomorphic section space $H^{0}(M, E)$ of $E$.

Theorem 1.1. Let $E$ be an Hermitian complex vector bundle or a Riemannian real vector bundle over a compact Hermitian manifold $(M, \omega)$ and $\nabla^{E}$ be any metric connection on $E$.

(1) If the second Hermitian-Ricci curvature $\operatorname{Tr}_{\omega} R^{E}$ is nonpositive everywhere, then every $\bar{\partial}_{E}$-closed section of $E$ is parallel, i.e. $\nabla^{E} s=0$;

(2) If the second Hermitian-Ricci curvature $\operatorname{Tr}_{\omega} R^{E}$ is nonpositive everywhere and negative at some point, then $\mathcal{H}_{\bar{\partial}_{E}}^{0}(M, E)=0$;

(3) If the second Hermitian-Ricci curvature $\operatorname{Tr}_{\omega} R^{E}$ is $p$-nonpositive everywhere and $p$-negative at some point, then $\mathcal{H}_{\bar{\partial}_{E}}\left(M, \Lambda^{q} E\right)=0$ for any $p \leq q \leq \operatorname{rank}(E)$.

The proof of this theorem is based on generalized Bochner-Kodaira identities on vector bundles over Hermitian manifolds (Theorem 4.5). We prove that (Theorem 4.8) the torsion integral of the Hermitian manifold can be killed if the background Hermitian metric $\omega$ is Gauduchon, i.e. $\partial \bar{\partial} \omega^{n-1}=0$. On the other hand, in the conformal class of any Hermitian metric, the Gauduchon metric always exists ([21]). So we can change the background metric in the conformal way and the positivity of the second Hermitian-Ricci curvature is preserved. This method is very useful on Hermitian manifolds. Kobayashi-Wu([31]) and Gauduchon([19]) obtained similar result in the special case when $\nabla^{E}$ is the Chern connection of the Hermitian holomorphic vector bundle $E$. Now we go back to the Hermitian manifold $(M, \omega)$.

Corollary 1.2. Let $(M, \omega)$ be a compact Hermitian manifold

(1) if the second Ricci-Chern curvature $\operatorname{Tr}_{\omega} \Theta$ is nonnegative everywhere and positive at some point, then $H_{\bar{\partial}}^{p, 0}(M)=0$ for any $1 \leq p \leq n$. In particular, the arithmetic genus $\chi(M, \mathcal{O})=1$;

(2) if the second Ricci-Chern curvature $\operatorname{Tr}_{\omega} \Theta$ is nonpositive everywhere and negative at some point, then the holomorphic vector bundle $\Lambda^{p} T^{1,0} M$ has no holomorphic vector field for any $1 \leq p \leq n$. 
Since the first Ricci-Chern curvature and the second Ricci-Chern curvature of an Hermitian manifold can not be compared, we can not derive that the manifold $M$ is Kähler, even if the second Ricci-Chern curvature is positive everywhere. In general, the first Ricci-Chern curvature is $d$-closed but the second Ricci-Chern curvature is not $d$-closed and so they are in the different $(d, \bar{\partial}, \partial)$-cohomology classes. For example, the Hopf manifold $\mathbb{S}^{2 n+1} \times \mathbb{S}^{1}$ with standard Hermitian metric has strictly positive second Ricci-Chern curvature and nonnegative first Ricci-Chern curvature, but it is non-Kähler. For more details, see Proposition 6.4.

Now we consider several special Hermitian manifolds. An interesting class of Hermitian manifolds is the balanced Hermitian manifolds, i.e., Hermitian manifolds with coclosed Kähler forms. It is well-known that every Kähler manifold is balanced. In some literatures, they are also called semi-Kähler manifolds. In complex dimension 1 and 2, every balanced Hermitian manifold is Kähler. However, in higher dimensions, there exist non-Kähler manifolds which admit balanced Hermitian metrics. Such examples were constructed by E. Calabi([7]), see also [23] and [36]. There are also some other important classes of non-Kähler balanced manifolds, such as: complex solvmanifolds, 1-dimensional families of Kähler manifolds (see [36]) and compact complex parallelizable manifolds (except complex torus) (see [46]). On the other hand, Alessandrini- Bassaneli( [2]) proved that every Moishezon manifold is balanced and so balanced manifolds can be constructed from Kähler manifolds by modification. For more examples, see [3], [36], [16] and [17].

Every balanced metric $\omega$ is a Gauduchon metric. In fact, $d^{*} \omega=0$ is equivalent to $d \omega^{n-1}=0$ and so $\partial \bar{\partial} \omega^{n-1}=0$. By [21], every Hermitian manifold has a Gauduchon metric. However, there are many manifolds which can not support balanced metrics. For example, the Hopf surface $\mathbb{S}^{3} \times \mathbb{S}^{1}$ is non-Kähler, so it has no balanced metric. For more discussion, one can see [7], [36],[42], [2] and [3].

On a compact balanced Hermitian manifold $M$, we can detect the holomorphic section spaces $H_{\bar{\partial}}^{p, 0}(M)$ by Levi-Civita connection. Let $\nabla$ be the complexified Levi-Civita connection and $\nabla^{\prime}, \nabla^{\prime \prime}$ the $(1,0)$ and $(0,1)$ components of $\nabla$ respectively. In general, holomorphic $p$ forms are not $\nabla^{\prime \prime}$-closed. The Ricci curvatures related to the Levi-Civita connection are defined in 2.11 and 2.29 .

Theorem 1.3. Let $(M, \omega)$ be a compact balanced Hermitian manifold. If the Hermitian-Ricci curvature $\left(R_{i \bar{j}}\right)$ of $M$ is nonnegative everywhere, then

(1) If $\varphi$ is a holomorphic $p$-form, then $\Delta_{\partial} \varphi=0$ and so $h^{p, 0}(M) \leq h^{0, p}(M)$ for any $1 \leq p \leq$ $n$;

(2) If the Hermitian-Ricci curvature $\left(R_{i \bar{j}}\right)$ is positive at some point, then $H_{\bar{\partial}}^{p, 0}(M)=0$ for any $1 \leq p \leq n$. In particular, the arithmetic genus $\chi(M, \mathcal{O})=1$.

The dual of Theorem 1.3 is

Theorem 1.4. Let $(M, \omega)$ be a compact balanced Hermitian manifold. If $2 \widehat{R}_{i \bar{j}}^{(2)}-R_{i \bar{j}}$ is nonpositive everywhere and negative at some point, there is no holomorphic vector field on $M$.

Remark 1.5. It is easy to see that the Hermitian-Ricci curvature tensor $\left(R_{i \bar{j}}\right)$ and second Ricci-Chern curvature tensor $\Theta^{(2)}:=\operatorname{Tr}_{\omega} \Theta$ can not be compared. Therefore, Theorem 1.3 and Corollary 1.2 are independent of each other. For the same reason, Theorem 1.4 and Corollary 1.2 are independent. Balanced Hermitian manifolds with nonnegative HermitianRicci curvatures are discussed in Proposition 3.5. 
As we discuss in the above, on Hermitian manifolds, the second Ricci curvature tensors of various metric connections are closely related to the geometry of Hermitian manifolds. A natural idea is to define a flow by using second Ricci curvature tensors of various metric connections. For example,

$$
\frac{\partial h}{\partial t}=-\Theta^{(2)}+\mu h, \quad \mu \in \mathbb{R}
$$

on a general Hermitian manifold $(M, h)$ by using the second Ricci-Chern curvature. This flow preserves the Kähler and the Hermitian structure and has short time solution on any compact Hermitian manifold. It is very similar to and closely related to the Hermitian Yang-Mills flow, the Kähler-Ricci flow and the harmonic map heat flow. It may be a bridge to connect them. In this paper we only briefly discuss its basic properties. In a subsequent paper([33]) we will study its geometric and analytic property in detail.

We would like to thank Yi Li, Jeffrey Streets, Valetino Tosatti for their useful comments on an earlier version of this paper.

\section{Various connections and curvatures on Hermitian manifolds}

\subsection{Complexified Riemannian curvature}

Let $(M, g)$ be a Riemannian manifold with Levi-Civita connection $\nabla$, the curvature $R$ of $(M, g, \nabla)$ is defined as

$$
R(X, Y, Z, W)=g\left(\left(\nabla_{X} \nabla_{Y}-\nabla_{Y} \nabla_{X}-\nabla_{[X, Y]}\right) Z, W\right)
$$

On an Hermitian manifold $(M, h)$, let $\nabla$ be the complexified Levi-Civita connection and $g$ the background Riemannian metric. Two metrics are related by

$$
d s_{h}^{2}=d s_{g}^{2}-\sqrt{-1} \omega_{h}
$$

where $\omega_{h}$ is the fundamental $(1,1)$-form (or Kähler form) associated to $h$. For any two holomorphic vector fields $X, Y \in \Gamma\left(M, T^{1,0} M\right)$,

$$
h(X, Y)=2 g(X, \bar{Y})
$$

This formula will be used in several definitions. In the local holomorphic coordinates $\left\{z^{1}, \cdots, z^{n}\right\}$ on $M$, the complexified Christoffel symbols are given by

$$
\Gamma_{A B}^{C}=\sum_{E} \frac{1}{2} g^{C E}\left(\frac{\partial g_{A E}}{\partial z^{B}}+\frac{\partial g_{B E}}{\partial z^{A}}-\frac{\partial g_{A B}}{\partial z^{E}}\right)=\sum_{E} \frac{1}{2} h^{C E}\left(\frac{\partial h_{A E}}{\partial z^{B}}+\frac{\partial h_{B E}}{\partial z^{A}}-\frac{\partial h_{A B}}{\partial z^{E}}\right)
$$

where $A, B, C, E \in\{1, \cdots, n, \overline{1}, \cdots, \bar{n}\}$ and $z^{A}=z^{i}$ if $A=i, z^{A}=\bar{z}^{i}$ if $A=\bar{i}$. For example

$$
\Gamma_{i j}^{k}=\frac{1}{2} h^{k \bar{\ell}}\left(\frac{\partial h_{j \bar{\ell}}}{\partial z^{i}}+\frac{\partial h_{i \bar{\ell}}}{\partial z^{j}}\right), \Gamma_{\bar{i} j}^{k}=\frac{1}{2} h^{k \bar{\ell}}\left(\frac{\partial h_{j \bar{\ell}}}{\partial \bar{z}^{i}}-\frac{\partial h_{j \bar{i}}}{\partial \bar{z}^{\ell}}\right)
$$

The complexified curvature components are

$$
\begin{aligned}
R_{A B C D}: & =2 \mathbf{g}\left(\left(\nabla_{\frac{\partial}{\partial z^{A}}} \nabla_{\frac{\partial}{\partial z^{B}}}-\nabla_{\frac{\partial}{\partial z^{B}}} \nabla_{\frac{\partial}{\partial z^{A}}}\right) \frac{\partial}{\partial z^{C}}, \frac{\partial}{\partial z^{D}}\right) \\
& =\mathbf{h}\left(\left(\nabla_{\frac{\partial}{\partial z^{A}}} \nabla_{\frac{\partial}{\partial z^{B}}}-\nabla_{\frac{\partial}{\partial z^{B}}} \nabla_{\frac{\partial}{\partial z^{A}}}\right) \frac{\partial}{\partial z^{C}}, \frac{\partial}{\partial z^{\bar{D}}}\right)
\end{aligned}
$$


Hence

$$
R_{A B C}^{D}=\sum_{E} R_{A B C E} h^{E D}=-\left(\frac{\partial \Gamma_{A C}^{D}}{\partial z^{B}}-\frac{\partial \Gamma_{B C}^{D}}{\partial z^{A}}+\Gamma_{A C}^{F} \Gamma_{F B}^{D}-\Gamma_{B C}^{F} \Gamma_{A F}^{D}\right)
$$

By the Hermitian property, we have, for example

$$
R_{i \bar{j} k}^{l}=-\left(\frac{\partial \Gamma_{i k}^{l}}{\partial \bar{z}^{j}}-\frac{\partial \Gamma_{\bar{j} k}^{l}}{\partial z^{i}}+\Gamma_{i k}^{s} \Gamma_{\bar{j} s}^{l}-\Gamma_{\bar{j} k}^{s} \Gamma_{i s}^{l}-\Gamma_{\bar{j} k}^{\bar{s}} \Gamma_{i \bar{s}}^{l}\right)
$$

Remark 2.1. We have $R_{A B C D}=R_{C D A B}$. In particular,

$$
R_{i \bar{j} k \bar{\ell}}=R_{k \bar{\ell} i \bar{j}}
$$

Unlike the Kähler case, we can define several Ricci curvatures:

Definition 2.2. (1) The complexified Ricci curvature on $(M, h)$ is defined by

$$
\mathscr{R}_{k \bar{\ell}}:=h^{i \bar{j}}\left(R_{k \bar{j} i \bar{\ell}}+R_{k i \bar{j} \ell}\right)
$$

The complexified scalar curvature of $h$ is defined as

$$
s_{h}:=h^{k \bar{\ell}} \mathscr{R}_{k \bar{\ell}}
$$

(2) The Hermitian-Ricci curvature is

$$
R_{k \bar{\ell}}:=h^{i \bar{j}} R_{i \bar{j} k \bar{\ell}}
$$

The Hermitian-scalar curvature of $h$ is given by

$$
S:=h^{k \bar{\ell}} R_{k \bar{\ell}}
$$

Lemma 2.3. On an Hermitian manifold,

$$
\overline{R_{A B C D}}=R_{\overline{A B C D}}, \quad \overline{\mathscr{R}_{k \bar{\ell}}}=\mathscr{R}_{\ell \bar{k}}, \quad \overline{R_{k \bar{\ell}}}=R_{\ell \bar{k}}
$$

and

$$
\mathscr{R}_{k \bar{\ell}}=h^{i \bar{j}}\left(2 R_{k \bar{j} i \bar{\ell}}-R_{k \bar{\ell} \bar{i} \bar{j}}\right)
$$

Proof. The Hermitian property of curvature tensors is obvious. By first Bianchi identity, we have

$$
R_{k i \overline{j \ell}}+R_{k \overline{j \ell} i}+R_{k \bar{\ell} \bar{i}}=0
$$

That is $R_{k i \bar{j} \bar{\ell}}=R_{k \bar{j} i \bar{\ell}}-R_{k \bar{\ell} i \bar{j}}$. The curvature formula 2.9 turns to be

$$
\mathscr{R}_{k \bar{\ell}}=h^{i \bar{j}}\left(2 R_{k \bar{j} \bar{\ell}}-R_{k \bar{\ell} i \bar{j}}\right)
$$

Definition 2.4. The Ricci curvatures are called positive ( resp. nonnegative, negative, nonpositive) if the corresponding Hermitian matrices are positive ( resp. nonnegative, negative, non-positive).

The following three formulas are used frequently in the sequel. 
Lemma 2.5. Assume $h_{i \bar{j}}=\delta_{i j}$ at a fixed point $p \in M$, we have the following formula

$$
\begin{aligned}
R_{i \bar{j} k \bar{\ell}}= & -\frac{1}{2}\left(\frac{\partial^{2} h_{i \bar{\ell}}}{\partial z^{k} \partial \bar{z}^{j}}+\frac{\partial^{2} h_{k \bar{j}}}{\partial z^{i} \partial \bar{z}^{\ell}}\right) \\
& +\frac{1}{4}\left(\frac{\partial h_{k \bar{q}}}{\partial z^{i}} \frac{\partial h_{q \bar{\ell}}}{\partial \bar{z}^{j}}+\frac{\partial h_{i \bar{q}}}{\partial z^{k}} \frac{\partial h_{q \bar{j}}}{\partial \bar{z}^{\ell}}\right)+\frac{1}{4}\left(\frac{\partial h_{i \bar{q}}}{\partial z^{k}} \frac{\partial h_{q \bar{\ell}}}{\partial \bar{z}^{j}}+\frac{\partial h_{k \bar{q}}}{\partial z^{i}} \frac{\partial h_{q \bar{j}}}{\partial \bar{z}^{\ell}}\right) \\
& +\frac{1}{4}\left(\frac{\partial h_{q \bar{\ell}}}{\partial z^{i}} \frac{\partial h_{k \bar{j}}}{\partial \bar{z}^{q}}+\frac{\partial h_{q \bar{j}}}{\partial z^{k}} \frac{\partial h_{i \bar{\ell}}}{\partial \bar{z}^{q}}\right)+\frac{1}{4}\left(\frac{\partial h_{i \bar{\ell}}}{\partial z^{q}} \frac{\partial h_{k \bar{q}}}{\partial \bar{z}^{j}}+\frac{\partial h_{k \bar{j}}}{\partial z^{q}} \frac{\partial h_{i \bar{q}}}{\partial \bar{z}^{\ell}}\right) \\
& -\frac{1}{4}\left(\frac{\partial h_{q \bar{\ell}}}{\partial z^{i}} \frac{\partial h_{k \bar{q}}}{\partial \bar{z}^{j}}+\frac{\partial h_{q \bar{j}}}{\partial z^{k}} \frac{\partial h_{i \bar{q}}}{\partial \bar{z}^{\ell}}\right)-\frac{1}{4}\left(\frac{\partial h_{i \bar{\ell}}}{\partial z^{q}} \frac{\partial h_{k \bar{j}}}{\partial \bar{z}^{q}}+\frac{\partial h_{k \bar{j}}}{\partial z^{q}} \frac{\partial h_{i \bar{\ell}}}{\partial \bar{z}^{q}}\right)
\end{aligned}
$$

By a linear transformation on the local holomorphic coordinates, one can get the following Lemma. For more details, we refer the reader to [44].

Lemma 2.6. Let $(M, h, \omega)$ be an Hermitian manifold. For any $p \in M$, there exist local holomorphic coordinates $\left\{z^{i}\right\}$ centered at a point $p$ such that

$$
h_{i \bar{j}}(p)=\delta_{i j} \quad \text { and } \quad \Gamma_{i j}^{k}(p)=0
$$

By Lemma 2.6, we have a simplified version of curvatures:

Lemma 2.7. Assume $h_{i \bar{j}}(p)=\delta_{i j}$ and $\Gamma_{i j}^{k}(p)=0$ at a fixed point $p \in M$,

$$
R_{i \bar{j} k \bar{\ell}}=-\frac{1}{2}\left(\frac{\partial^{2} h_{i \bar{\ell}}}{\partial z^{k} \partial \bar{z}^{j}}+\frac{\partial^{2} h_{k \bar{j}}}{\partial z^{i} \partial \bar{z}^{\ell}}\right)-\sum_{q}\left(\frac{\partial h_{q \bar{\ell}}}{\partial z^{i}} \frac{\partial h_{k \bar{q}}}{\partial \bar{z}^{j}}+\frac{\partial h_{q \bar{j}}}{\partial z^{k}} \frac{\partial h_{i \bar{q}}}{\partial \bar{z}^{\ell}}\right)
$$

For Hermitian-Ricci curvatures

$$
R_{k \bar{\ell}}=h^{i \bar{j}} R_{i \bar{j} k \bar{\ell}}=-\frac{1}{2} \sum_{s}\left(\frac{\partial^{2} h_{s \bar{\ell}}}{\partial z^{k} \partial \bar{z}^{s}}+\frac{\partial^{2} h_{k \bar{s}}}{\partial z^{s} \partial \bar{z}^{\ell}}\right)-\sum_{q, s}\left(\frac{\partial h_{q \bar{\ell}}}{\partial z^{s}} \frac{\partial h_{k \bar{q}}}{\partial \bar{z}^{s}}+\frac{\partial h_{k \bar{q}}}{\partial z^{s}} \frac{\partial h_{q \bar{\ell}}}{\partial \bar{z}^{s}}\right)
$$

and

$$
h^{i \bar{j}} R_{k \bar{j} i \bar{\ell}}=h^{i \bar{j}} R_{i \bar{\ell} k \bar{j}}=-\frac{1}{2} \sum_{s}\left(\frac{\partial^{2} h_{k \bar{\ell}}}{\partial z^{s} \partial \bar{z}^{s}}+\frac{\partial^{2} h_{s \bar{s}}}{\partial z^{k} \partial \bar{z}^{\ell}}\right)-\sum_{q, s}\left(\frac{\partial h_{q \bar{\ell}}}{\partial z^{k}} \frac{\partial h_{s \bar{q}}}{\partial \bar{z}^{s}}+\frac{\partial h_{q \bar{s}}}{\partial z^{s}} \frac{\partial h_{k \bar{q}}}{\partial \bar{z}^{\ell}}\right)
$$

For complexified Ricci curvature,

$$
\begin{aligned}
\mathscr{R}_{k \bar{\ell}} & =\frac{1}{2} \sum_{s}\left(\frac{\partial^{2} h_{s \bar{\ell}}}{\partial z^{k} \partial \bar{z}^{s}}+\frac{\partial^{2} h_{k \bar{s}}}{\partial z^{s} \partial \bar{z}^{\ell}}\right)-\sum_{s}\left(\frac{\partial^{2} h_{k \bar{\ell}}}{\partial z^{s} \partial \bar{z}^{s}}+\frac{\partial^{2} h_{s \bar{s}}}{\partial z^{k} \partial \bar{z}^{\ell}}\right) \\
& +\sum_{q, s}\left(\frac{\partial h_{q \bar{\ell}}}{\partial z^{s}} \frac{\partial h_{k \bar{q}}}{\partial \bar{z}^{s}}+\frac{\partial h_{k \bar{q}}}{\partial z^{s}} \frac{\partial h_{q \bar{\ell}}}{\partial \bar{z}^{s}}\right)-2 \sum_{q, s}\left(\frac{\partial h_{q \bar{\ell}}}{\partial z^{k}} \frac{\partial h_{s \bar{q}}}{\partial \bar{z}^{s}}+\frac{\partial h_{q \bar{s}}}{\partial z^{s}} \frac{\partial h_{k \bar{q}}}{\partial \bar{z}^{\ell}}\right)
\end{aligned}
$$

\subsection{Curvature of complexified Levi-Civita connection on $T^{1,0} M$}

Since $T^{1,0} M$ is a subbundle of $T_{\mathbb{C}} M$, there is an induced connection $\widehat{\nabla}$ on $T^{1,0} M$ given by

$$
\widehat{\nabla}=\pi \circ \nabla: T^{1,0} M \stackrel{\nabla}{\rightarrow} \Gamma\left(M, T_{\mathbb{C}} M \otimes T_{\mathbb{C}} M\right) \stackrel{\pi}{\rightarrow} \Gamma\left(M, T_{\mathbb{C}} M \otimes T^{1,0} M\right)
$$

The curvature $\widehat{R} \in \Gamma\left(M, \Lambda^{2} T_{\mathbb{C}} M \otimes T^{* 1,0} M \otimes T^{1,0} M\right)$ of $\widehat{\nabla}$ is given by

$$
\widehat{R}(X, Y) s=\widehat{\nabla}_{X} \widehat{\nabla}_{Y} s-\widehat{\nabla}_{Y} \widehat{\nabla}_{X} s-\widehat{\nabla}_{[X, Y]} s
$$


for any $X, Y \in T_{\mathbb{C}} M$ and $s \in T^{1,0} M$. It has components

$$
\widehat{R}_{A B k}^{l}=\frac{\partial \Gamma_{B k}^{l}}{\partial z^{A}}-\frac{\partial \Gamma_{A k}^{l}}{\partial z^{B}}-\Gamma_{A k}^{s} \Gamma_{B s}^{l}+\Gamma_{B k}^{s} \Gamma_{A s}^{l}
$$

where

$$
\widehat{R}\left(\frac{\partial}{\partial z^{A}}, \frac{\partial}{\partial z^{B}}\right) \frac{\partial}{\partial z^{k}}=\sum_{l} \widehat{R}_{A B k}^{l} \frac{\partial}{\partial z^{\ell}}
$$

For example,

$$
\widehat{R}_{i \bar{j} k}^{l}=-\left(\frac{\partial \Gamma_{i k}^{l}}{\partial \bar{z}^{j}}-\frac{\partial \Gamma_{\bar{j} k}^{l}}{\partial z^{i}}+\Gamma_{i k}^{s} \Gamma_{\bar{j} s}^{l}-\Gamma_{\bar{j} k}^{s} \Gamma_{s i}^{l}\right)
$$

With respect to the Hermitian metric $h$ on $T^{1,0} M$, we can define

$$
\widehat{R}_{A B k \bar{l}}=\sum_{s=1}^{n} \widehat{R}_{A B k}^{s} h_{s \bar{\ell}}
$$

Definition 2.8. The first Ricci curvature of the Hermitian vector bundle $\left(T^{1,0} M, \widehat{\nabla}\right)$ is defined by

$$
\widehat{R}_{i \bar{j}}^{(1)}=h^{k \bar{\ell}} \widehat{R}_{i \bar{j} k \bar{\ell}}
$$

The second Ricci curvature of it is

$$
\widehat{R}_{k \bar{\ell}}^{(2)}=h^{i \bar{j}} \widehat{R}_{i \bar{j} k \bar{\ell}}
$$

The scalar curvature of $\widehat{\nabla}$ on $T^{1,0} M$ is denoted by

$$
S^{L C}=h^{i \bar{j}} h^{k \bar{\ell}} \widehat{R}_{i \bar{j} k \bar{\ell}}
$$

By Lemma 2.6, we have the following formulas

Lemma 2.9. On an Hermitian manifold $(M, h)$, on a point $p$ with $h_{i \bar{j}}(p)=\delta_{i j}$ and $\Gamma_{i j}^{k}(p)=$ 0 ,

$$
\widehat{R}_{i \bar{j} k \bar{\ell}}=-\frac{1}{2}\left(\frac{\partial^{2} h_{i \bar{\ell}}}{\partial z^{k} \partial \bar{z}^{j}}+\frac{\partial^{2} h_{k \bar{j}}}{\partial z^{i} \partial \bar{z}^{\ell}}\right)-\sum_{q} \frac{\partial h_{q \bar{\ell}}}{\partial z^{i}} \frac{\partial h_{k \bar{q}}}{\partial \bar{z}^{j}}
$$

For the Ricci curvatures,

$$
\widehat{R}_{i \bar{j}}^{(1)}=-\frac{1}{2} \sum_{k}\left(\frac{\partial^{2} h_{i \bar{k}}}{\partial z^{k} \partial \bar{z}^{j}}+\frac{\partial^{2} h_{k \bar{j}}}{\partial z^{i} \partial \bar{z}^{k}}\right)-\sum_{k, q} \frac{\partial h_{q \bar{k}}}{\partial z^{i}} \frac{\partial h_{k \bar{q}}}{\partial \bar{z}^{j}}
$$

and

$$
\widehat{R}_{i \bar{j}}^{(2)}=-\frac{1}{2} \sum_{k}\left(\frac{\partial^{2} h_{i \bar{k}}}{\partial z^{k} \partial \bar{z}^{j}}+\frac{\partial^{2} h_{k \bar{j}}}{\partial z^{i} \partial \bar{z}^{k}}\right)-\sum_{k, q} \frac{\partial h_{i \bar{q}}}{\partial \bar{z}^{k}} \frac{\partial h_{q \bar{j}}}{\partial z^{k}}
$$

Moreover,

$$
\widehat{R}_{i \bar{j}}^{(1)}-\widehat{R}_{i \bar{j}}^{(2)}=h_{m \bar{j}} h^{\ell \bar{k}} \Gamma_{\bar{k} i}^{\bar{q}} \Gamma_{\ell \bar{q}}^{m}-\Gamma_{k \bar{j}}^{\bar{q}} \Gamma_{i \bar{q}}^{k}=\sum_{k, q}\left(\frac{\partial h_{i \bar{q}}}{\partial \bar{z}^{k}} \frac{\partial h_{q \bar{j}}}{\partial z^{k}}-\frac{\partial h_{i \bar{q}}}{\partial z^{k}} \frac{\partial h_{q \bar{j}}}{\partial \bar{z}^{k}}\right)
$$




\subsection{Curvature of Chern connection on $T^{1,0} M$}

On the Hermitian holomorphic vector bundle $\left(T^{1,0} M, h\right)$, the Chern connection $\nabla^{C H}$ is the unique connection which is compatible with the complex structure and the Hermitian metric. Its curvature components are

$$
\Theta_{i \bar{j} k \bar{\ell}}=-\frac{\partial^{2} h_{k \bar{\ell}}}{\partial z^{i} \partial \bar{z}^{j}}+h^{p \bar{q}} \frac{\partial h_{p \bar{\ell}}}{\partial \bar{z}^{j}} \frac{\partial h_{k \bar{q}}}{\partial z^{i}}
$$

It is well-known that the first Ricci-Chern curvature

$$
\Theta^{(1)}:=\frac{\sqrt{-1}}{2 \pi} \Theta_{i \bar{j}}^{(1)} d z^{i} \wedge d \bar{z}^{j}
$$

represents the first Chern class of $M$ where

$$
\Theta_{i \bar{j}}^{(1)}=h^{k \bar{\ell}} \Theta_{i \bar{j} k \bar{\ell}}=-\frac{\partial^{2} \log \operatorname{det}\left(h_{k \bar{\ell}}\right)}{\partial z^{i} \partial \bar{z}^{j}}
$$

The second Ricci-Chern curvature components are

$$
\Theta_{i \bar{j}}^{(2)}=h^{k \bar{\ell}} \Theta_{k \bar{\ell} i \bar{j}}
$$

The scalar curvature of the Chern connection is defined by

$$
S^{C H}=h^{i \bar{j}} h^{k \bar{\ell}} \Theta_{i \bar{j} k \bar{\ell}}
$$

\subsection{Curvature of Bismut connection on $T^{1,0} M$}

In [4], Bismut defined a class of connections on Hermitian manifolds. In this subsection, we choose one of them (see [35], p. 21). The Bismut connection $\nabla^{B}$ on the holomorphic tangent bundle $\left(T^{1,0} M, h\right)$ is characterized by

$$
\nabla^{B}=\nabla+S^{B}
$$

where $S^{B}$ is 1 -form with values in $\operatorname{End}\left(T^{1,0} M\right)$

$$
\mathbf{h}\left(S^{B}(X) Y, Z\right)=2 \mathbf{g}\left(S^{B}(X) Y, \bar{Z}\right)=\sqrt{-1}(\partial-\bar{\partial}) \omega_{h}(X, Y, \bar{Z})
$$

for any $Y, Z \in T^{1,0} M$ and $X \in T_{\mathbb{C}} M$. Let $\widetilde{\Gamma}_{i \alpha}^{\beta}$ and $\widetilde{\Gamma}_{\bar{j} \alpha}^{\beta}$ be the Christoffel symbols of the Bismut connection where $i, j, \alpha, \beta \in\{1, \cdots, n\}$. We use different types of letters since the Bismut connection is not torsion free.

Lemma 2.10. We have the following relations between $\widetilde{\Gamma}$ and $\Gamma$,

$$
\widetilde{\Gamma}_{i \alpha \bar{\beta}}:=h_{\beta \bar{\gamma}} \Gamma_{i \alpha}^{\bar{\gamma}}=\Gamma_{i \alpha \bar{\beta}}+\Gamma_{\alpha \bar{\beta} i}=\frac{\partial h_{i \bar{\beta}}}{\partial z^{\alpha}}, \quad \widetilde{\Gamma}_{\bar{j} \alpha \bar{\beta}}=2 \Gamma_{\bar{j} \alpha \bar{\beta}}
$$

Proof. Let $X=\frac{\partial}{\partial z^{i}}, Y=\frac{\partial}{\partial z^{j}}, Z=\frac{\partial}{\partial z^{k}}$. Since $\omega_{h}=\frac{\sqrt{-1}}{2} h_{m \bar{n}} d z^{m} \wedge d \bar{z}^{n}$, we obtain

$$
\begin{aligned}
\sqrt{-1}(\partial-\bar{\partial}) \omega_{h}(X, Y, \bar{Z}) & =-\frac{1}{2} \frac{\partial h_{m \bar{n}}}{\partial z^{p}} d z^{p} d z^{m} d \bar{z}^{n}\left(\frac{\partial}{\partial z^{i}}, \frac{\partial}{\partial z^{j}}, \frac{\partial}{\partial \bar{z}^{k}}\right) \\
& =\frac{1}{2}\left(\frac{\partial h_{i \bar{k}}}{\partial z^{j}}-\frac{\partial h_{j \bar{k}}}{\partial z^{i}}\right) \\
& =\Gamma_{j \bar{k}}^{\bar{s}} h_{i \bar{s}}=\Gamma_{j \bar{k} i}
\end{aligned}
$$


On the other hand

$$
h\left(\nabla_{\frac{\partial}{\partial z^{i}}}^{B} \frac{\partial}{\partial z^{j}}, \frac{\partial}{\partial z^{k}}\right)=\widetilde{\Gamma}_{i j \bar{k}}
$$

Using the definition of Bismut connection, we get

$$
\widetilde{\Gamma}_{i \alpha \bar{\beta}}=\Gamma_{i \alpha \bar{\beta}}+\Gamma_{\alpha \bar{\beta} i}=\frac{\partial h_{i \bar{\beta}}}{\partial z^{\alpha}}
$$

The proof of the other one is similar.

The Bismut curvature $B \in \Gamma\left(M, \Lambda^{1,1} T^{*} M \otimes \operatorname{End}\left(T^{1,0} M\right)\right)$ is given by

$$
B_{i \bar{j} \alpha}^{\beta}=-\frac{\partial \widetilde{\Gamma}_{i \alpha}^{\beta}}{\partial \bar{z}^{j}}+\frac{\partial \widetilde{\Gamma}_{\bar{j} \alpha}^{\beta}}{\partial z^{i}}-\widetilde{\Gamma}_{i \alpha}^{\gamma} \widetilde{\Gamma}_{\bar{j} \gamma}^{\beta}+\widetilde{\Gamma}_{\bar{j} \alpha}^{\gamma} \widetilde{\Gamma}_{i \gamma}^{\beta}
$$

Lemma 2.11. Assume $h_{i \bar{j}}(p)=\delta_{i j}$ and $\Gamma_{i j}^{k}(p)=0$ at a fixed point $p \in M$,

$$
B_{i \bar{j} \alpha \bar{\beta}}=-\left(\frac{\partial^{2} h_{i \bar{\beta}}}{\partial \bar{z}^{j} \partial z^{\alpha}}+\frac{\partial^{2} h_{\alpha \bar{j}}}{\partial z^{i} \partial \bar{z}^{\beta}}-\frac{\partial^{2} h_{\alpha \bar{\beta}}}{\partial z^{i} \partial \bar{z}^{j}}\right)+\sum_{\gamma} \frac{\partial h_{\alpha \bar{\gamma}}}{\partial z^{i}} \frac{\partial h_{\gamma \bar{\beta}}}{\partial \bar{z}^{j}}-4 \sum_{\gamma} \frac{\partial h_{\alpha \bar{\gamma}}}{\partial \bar{z}^{j}} \frac{\partial h_{\gamma \bar{\beta}}}{\partial z^{i}}
$$

Proof. It follows by 2.42 and 2.45 .

We can define the first Ricci-Bismut curvature $B_{i \bar{j}}^{(1)}$, the second Ricci-Bismut curvature $B_{i \bar{j}}^{(2)}$ and scalar curvature $S^{B M}$ similarly.

\subsection{Relations among the four curvatures on Hermitian manifolds}

Proposition 2.12. On an Hermitian manifold $(M, h)$, we have

$$
R_{i j k \bar{l}}=\widehat{R}_{i j k \bar{\ell}}, \quad R_{\overline{i j k} \bar{\ell}}=\widehat{R}_{\overline{i j k} \bar{\ell}}
$$

and for any $u, v \in \mathbb{C}^{n}$,

$$
\left(R_{i \bar{j} k \bar{\ell}}-\widehat{R}_{i \bar{j} k \bar{\ell}}\right) u^{i} \bar{u}^{j} v^{k} \bar{v}^{\ell} \leq 0
$$

In particular, $R_{i \bar{j}} \leq \widehat{R}_{i \bar{j}}^{(1)}$ and $R_{i \bar{j}} \leq \widehat{R}_{i \bar{j}}^{(2)}$ in the sense of Hermitian matrices.

Proof. Let

$$
T_{i \bar{j} k \bar{\ell}}=R_{i \bar{j} k \bar{\ell}}-\widehat{R}_{i \bar{j} k \bar{\ell}}=\Gamma_{\bar{j} k}^{\bar{s}} \Gamma_{i \bar{s}}^{t} h_{t \bar{\ell}}
$$

Without loss generality, we assume $h_{i \bar{j}}=\delta_{i j}$ at a fixed point, then

$$
T_{i \bar{j} k \bar{\ell}}=\sum_{s} \Gamma_{\bar{j} k s} \Gamma_{i \bar{s} \bar{\ell}}=-\sum_{s} \Gamma_{i \bar{s} \bar{\ell}} \overline{\Gamma_{j \bar{s} \bar{k}}}
$$

where

$$
\Gamma_{i \bar{s} \bar{\ell}}=\frac{1}{2}\left(\frac{\partial h_{i \bar{\ell}}}{\partial \bar{z}^{s}}-\frac{\partial h_{i \bar{s}}}{\partial \bar{z}^{\ell}}\right)=-\Gamma_{i \bar{\ell} \bar{s}}
$$

and so $T_{i \bar{j} k \bar{\ell}} u^{i} \bar{u}^{j} v^{k} \bar{v}^{\ell} \leq 0$.

Remark 2.13. (1) Because of the second order terms in $R, \widehat{R}, \Theta$ and $B$, we can not compare $R, \widehat{R}$ with $\Theta, B$.

(2) Since the third order terms of $\partial \Theta^{(2)}$ are not zero in general. Therefore it is possible that $\Theta^{(1)}$ and $\Theta^{(2)}$ are not in the same $(d, \partial, \bar{\partial})$-cohomology class. For the same reason $B^{(1)}$ and $B^{(2)}$ are not in the same $(d, \partial, \bar{\partial})$-cohomology class.

(3) If the manifold $(M, h)$ is Kähler, then all curvatures are the same. 


\section{Curvature relations on special Hermitian manifolds}

\subsection{Curvatures relations on balanced Hermitian manifolds}

The following lemma is well-known( for example [18]), and we include a proof here in our setting.

Lemma 3.1. Let $(M, \omega)$ be a compact Hermitian manifold. The following conditions are equivalent:

(1) $d^{*} \omega=0$;

(2) $d \omega^{n-1}=0$;

(3) For any smooth function $f \in C^{\infty}(M)$,

$$
\frac{1}{2} \Delta_{d} f=\Delta_{\bar{\partial}} f=\Delta_{\partial} f=-h^{i \bar{j}} \frac{\partial^{2} f}{\partial z^{i} \partial \bar{z}^{j}}
$$

(4) $\Gamma_{i \ell}^{\ell}=0$ for any $1 \leq i \leq n$.

Proof. On a compact Hermitian manifold, $d^{*} \omega=-* d * \omega=-c_{n} * d \omega^{n-1}$ where $c_{n}$ is a constant depending only on the complex dimension $n$ of $M$. On the other hand, the Hodge * is an isomorphism, and so (1) and (2) are equivalent. If $f$ is a smooth function on $M$,

$$
\left\{\begin{array}{l}
\Delta_{\bar{\partial}} f=-h^{i \bar{j}} \frac{\partial^{2} f}{\partial z^{i} \partial \bar{z}^{j}}+2 h^{i \bar{j}} \Gamma_{i \bar{\jmath}}^{\bar{\ell}} \frac{\partial f}{\partial \bar{z}^{\ell}} \\
\Delta_{\partial} f=-h^{i \bar{j}} \frac{\partial^{2} f}{\partial z^{i} \partial \bar{z}^{j}}+2 h^{i \bar{j}} \Gamma_{\bar{j} i}^{k} \frac{\partial f}{\partial z^{k}}
\end{array}\right.
$$

On the other hand,

$$
h^{i \bar{j}} \Gamma_{i \bar{j}}^{\bar{\ell}}=-\Gamma_{k \bar{j}}^{\bar{j}} h^{k \bar{\ell}} \text { and } h^{i \bar{j}} \Gamma_{\bar{j} i}^{k}=-\Gamma_{\bar{\ell} i}^{i} h^{k \bar{\ell}}
$$

Therefore (3) and (4) are equivalent. For the equivalence of (1) and (4), see Lemma 8.8.

Definition 3.2. An Hermitian manifold $(M, \omega)$ is called balanced if it satisfies one of the conditions in Lemma 3.1.

On a balanced Hermitian manifold, there are more symmetries on the second derivatives of the metric.

Lemma 3.3. Let $(M, h)$ be a balanced Hermitian manifold. On a point $p$ with $h_{i \bar{j}}(p)=\delta_{i j}$ and $\Gamma_{i j}^{k}(p)=0$,

$$
\sum_{s} \frac{\partial h_{s \bar{i}}}{\partial \bar{z}^{s}}=\sum_{s} \frac{\partial h_{s \bar{s}}}{\partial \bar{z}^{i}}=0
$$

and

$$
\sum_{i} \frac{\partial^{2} h_{i \bar{\ell}}}{\partial z^{k} \partial \bar{z}^{i}}=\sum_{i} \frac{\partial^{2} h_{k \bar{i}}}{\partial z^{i} \partial \bar{z}^{\ell}}=\sum_{i} \frac{\partial^{2} h_{i \bar{i}}}{\partial z^{k} \partial \bar{z}^{\ell}}-2 \sum_{i, q} \frac{\partial h_{q \bar{\ell}}}{\partial \bar{z}^{i}} \frac{\partial h_{k \bar{q}}}{\partial z^{i}}
$$

Proof. At a fixed point $p$, if $h_{i \bar{j}}=0$ and $\Gamma_{i j}^{k}=0$, then

$$
\frac{\partial h_{i \bar{j}}}{\partial \bar{z}^{k}}=-\frac{\partial h_{i \bar{k}}}{\partial \bar{z}^{j}}
$$


The balanced condition $\sum_{s} \Gamma_{\bar{i} s}^{s}=0$ is reduced to

$$
\sum_{s} \frac{\partial h_{s \bar{s}}}{\partial \bar{z}^{i}}=\sum_{s} \frac{\partial h_{s \bar{i}}}{\partial \bar{z}^{s}}=0
$$

by formula 3.6. By the balanced condition

$$
\begin{aligned}
0=\frac{\partial \Gamma_{\bar{\ell} i}^{i}}{\partial z^{k}} & =\frac{\partial}{\partial z^{k}}\left(\frac{1}{2} h^{i \bar{q}}\left(\frac{\partial h_{i \bar{q}}}{\partial \bar{z}^{\ell}}-\frac{\partial h_{i \bar{\ell}}}{\partial \bar{z}^{q}}\right)\right) \\
& =\frac{1}{2} \sum_{i}\left(\frac{\partial^{2} h_{i \bar{i}}}{\partial z^{k} \partial \bar{z}^{\ell}}-\frac{\partial^{2} h_{\bar{i} \bar{\ell}}}{\partial z^{k} \partial \bar{z}^{i}}\right)-\sum_{i, q} \frac{\partial h_{q \bar{\ell}}}{\partial \bar{z}^{i}} \frac{\partial h_{k \bar{q}}}{\partial z^{i}}
\end{aligned}
$$

Hence, we obtain formula 3.5.

Proposition 3.4. Let $(M, h)$ be a balanced Hermitian manifold. At a point $p$ with $h_{i \bar{j}}(p)=\delta_{i j}$ and $\Gamma_{i j}^{k}(p)=0$, we have following formulas about various Ricci curvatures:

$$
\begin{aligned}
\Theta_{k \bar{\ell}}^{(1)} & =\widehat{R}_{k \bar{\ell}}^{(1)}=B_{k \bar{\ell}}^{(1)}=-\sum_{i} \frac{\partial^{2} h_{i \bar{i}}}{\partial z^{k} \partial \bar{z}^{\ell}}+\sum_{q, i} \frac{\partial h_{q \bar{\ell}}}{\partial \bar{z}^{i}} \frac{\partial h_{k \bar{q}}}{\partial z^{i}} \\
\Theta_{k \bar{\ell}}^{(2)} & =-\sum_{i} \frac{\partial^{2} h_{k \bar{\ell}}}{\partial z^{i} \partial \bar{z}^{i}}+\sum_{i, q} \frac{\partial h_{q \bar{\ell}}}{\partial \bar{z}^{i}} \frac{\partial h_{k \bar{q}}}{\partial z^{i}} \\
\widehat{R}_{k \bar{\ell}}^{(2)} & =-\sum_{i} \frac{\partial^{2} h_{i \bar{i}}}{\partial z^{k} \partial \bar{z}^{\ell}}+\sum_{i, q}\left(2 \frac{\partial h_{q \bar{\ell}}}{\partial \bar{z}^{i}} \frac{\partial h_{k \bar{q}}}{\partial z^{i}}-\frac{\partial h_{k \bar{q}}}{\partial \bar{z}^{i}} \frac{\partial h_{q \bar{\ell}}}{\partial z^{i}}\right) \\
B_{k \bar{\ell}}^{(2)} & =-\sum_{i} \frac{\partial^{2} h_{i \bar{i}}}{\partial z^{k} \partial \bar{z}^{\ell}}+\sum_{i, q}\left(5 \frac{\partial h_{q \bar{\ell}}}{\partial \bar{z}^{i}} \frac{\partial h_{k \bar{q}}}{\partial z^{i}}-4 \frac{\partial h_{k \bar{q}}}{\partial \bar{z}^{i}} \frac{\partial h_{q \bar{\ell}}}{\partial z^{i}}\right) \\
R_{k \bar{\ell}} & =-\sum_{i} \frac{\partial^{2} h_{i \bar{i}}}{\partial z^{k} \partial \bar{z}^{\ell}}+\sum_{i, q}\left(\frac{\partial h_{q \bar{\ell}}}{\partial \bar{z}^{i}} \frac{\partial h_{k \bar{q}}}{\partial z^{i}}-\frac{\partial h_{k \bar{q}}}{\partial \bar{z}^{i}} \frac{\partial h_{q \bar{\ell}}}{\partial z^{i}}\right) \\
\mathscr{R}_{k \bar{\ell}} & =-\sum_{i} \frac{\partial^{2} h_{k \bar{\ell}}}{\partial z^{i} \partial \bar{z}^{i}}-\sum_{i, q}\left(\frac{\partial h_{q \bar{\ell}}}{\partial \bar{z}^{i}} \frac{\partial h_{k \bar{q}}}{\partial z^{i}}-\frac{\partial h_{k \bar{q}}}{\partial \bar{z}^{i}} \frac{\partial h_{q \bar{\ell}}}{\partial z^{i}}\right)
\end{aligned}
$$

Proof. In 2.32, 2.33, 2.37,2.38, 2.19, 2.21, we get expressions for all Ricci curvatures on Hermitian manifolds. By balanced relations 3.4 and 3.5, we get simplified versions of all Ricci curvatures.

Proposition 3.5. (1) A balanced Hermitian manifold with positive Hermitian-Ricci curvature $R_{i \bar{j}}$ is Kähler.

(2) Let $(M, h)$ be a compact balanced Hermitian manifold. If the Hermitian-Ricci curvature is nonnegative everywhere and positive at some point, then $M$ is Moishezon.

Proof. (1) On a balanced Hermitian manifold

$$
\Theta_{i \bar{j}}^{(1)}=\widehat{R}_{i \bar{j}}^{(1)} \geq R_{i \bar{j}}
$$

If $R_{i \bar{j}}$ is Hermitian positive, then $\Theta_{i \bar{j}}^{(1)}$ is Hermitian positive, and so

$$
\Omega=-\frac{\sqrt{-1}}{2 \pi} \partial \bar{\partial} \log \operatorname{det}\left(h_{k \bar{\ell}}\right)
$$


is a Kähler metric.

(2) If the Hermitian-Ricci curvature is nonnegative everywhere and positive at some point, so is $\Theta_{i \bar{j}}^{(1)}$. The Hermitian line bundle $L=\operatorname{det}\left(T^{1,0} M\right)$ satisfies

$$
\int_{M} c_{1}(L)^{n}>0
$$

By Siu-Demailly's solution of Grauert-Riemenschneider conjecture ([39] [9]), $M$ is Moishezon.

\subsection{Curvature relations on Hermitian manifolds with $\Lambda(\partial \bar{\partial} \omega)=0$}

Now we consider a compact Hermitian manifold $(M, \omega)$ with $\Lambda(\partial \bar{\partial} \omega)=0$. The condition $\Lambda(\partial \bar{\partial} \omega)=0$ is equivalent to

$$
\sum_{k}\left(\frac{\partial h_{i \bar{j}}}{\partial z^{k} \partial \bar{z}^{k}}+\frac{\partial h_{k \bar{k}}}{\partial z^{i} \partial \bar{z}^{j}}\right)=\sum_{k}\left(\frac{\partial h_{i \bar{k}}}{\partial z^{k} \partial \bar{z}^{j}}+\frac{\partial^{2} h_{k \bar{j}}}{\partial z^{i} \partial \bar{z}^{k}}\right)
$$

for any $i, j$. We can use 3.17 to simplify Ricci curvatures and get relations among them.

Proposition 3.6. Let $(M, h)$ be a compact Hermitian manifold with $\Lambda(\partial \bar{\partial} \omega)=0$. At a point $p$ with $h_{i \bar{j}}(p)=\delta_{i j}$ and $\Gamma_{i j}^{k}(p)=0$, the following identities about Ricci curvatures hold:

$$
\begin{aligned}
\Theta_{k \bar{\ell}}^{(1)}= & -\sum_{i} \frac{\partial^{2} h_{i \bar{i}}}{\partial z^{k} \partial \bar{z}^{\ell}}+\sum_{q, i} \frac{\partial h_{q \bar{\ell}}}{\partial \bar{z}^{i}} \frac{\partial h_{k \bar{q}}}{\partial z^{i}} \\
\Theta_{k \bar{\ell}}^{(2)}= & -\sum_{i} \frac{\partial^{2} h_{k \bar{\ell}}}{\partial z^{i} \partial \bar{z}^{i}}+\sum_{i, q} \frac{\partial h_{q \bar{\ell}}}{\partial \bar{z}^{i}} \frac{\partial h_{k \bar{q}}}{\partial z^{i}} \\
\widehat{R}_{k \bar{\ell}}^{(1)}= & -\frac{1}{2} \sum_{i}\left(\frac{\partial^{2} h_{k \bar{\ell}}}{\partial z^{i} \partial \bar{z}^{i}}+\frac{\partial^{2} h_{i \bar{i}}}{\partial z^{k} \partial \bar{z}^{\ell}}\right)-\sum_{i, q} \frac{\partial h_{q \bar{\ell}}}{\partial \bar{z}^{i}} \frac{\partial h_{k \bar{q}}}{\partial z^{i}} \\
\widehat{R}_{k \bar{\ell}}^{(2)}= & -\frac{1}{2} \sum_{i}\left(\frac{\partial^{2} h_{k \bar{\ell}}}{\partial z^{i} \partial \bar{z}^{i}}+\frac{\partial^{2} h_{i \bar{i}}}{\partial z^{k} \partial \bar{z}^{\ell}}\right)-\sum_{i, q} \frac{\partial h_{k \bar{q}}}{\partial \bar{z}^{i}} \frac{\partial h_{q \bar{\ell}}}{\partial z^{i}} \\
B_{k \bar{\ell}}^{(1)}= & -\sum_{i} \frac{\partial^{2} h_{k \bar{\ell}}}{\partial z^{i} \partial \bar{z}^{i}}+\sum_{i, q}\left(\frac{\partial h_{q \bar{\ell}}}{\partial \bar{z}^{i}} \frac{\partial h_{k \bar{q}}}{\partial z^{i}}-4 \frac{\partial h_{q \bar{\ell}}}{\partial z^{i}} \frac{\partial h_{k \bar{q}}}{\partial \bar{z}^{i}}\right) \\
B_{k \bar{\ell}}^{(2)}= & -\sum_{i} \frac{\partial^{2} h_{i \bar{i}}}{\partial z^{k} \partial \bar{z}^{\ell}}+\sum_{i, q}\left(\frac{\partial h_{q \bar{\ell}}}{\partial \bar{z}^{i}} \frac{\partial h_{k \bar{q}}}{\partial z^{i}}-4 \frac{\partial h_{k \bar{q}}}{\partial \bar{z}^{i}} \frac{\partial h_{q \bar{\ell}}}{\partial z^{i}}\right) \\
R_{k \bar{\ell}}= & -\frac{1}{2} \sum_{i}\left(\frac{\partial^{2} h_{k \bar{\ell}}}{\partial z^{i} \partial \bar{z}^{i}}+\frac{\partial^{2} h_{i \bar{i}}}{\partial z^{k} \partial \bar{z}^{\ell}}\right)-\sum_{i, q}\left(\frac{\partial h_{q \bar{\ell}}}{\partial \bar{z}^{i}} \frac{\partial h_{k \bar{q}}}{\partial z^{i}}+\frac{\partial h_{k \bar{q}}}{\partial \bar{z}^{i}} \frac{\partial h_{q \bar{\ell}}}{\partial z^{i}}\right) \\
\mathscr{R}_{k \bar{\ell}}= & -\frac{1}{2} \sum_{i}\left(\frac{\partial^{2} h_{k \bar{\ell}}}{\partial z^{i} \partial \bar{z}^{i}}+\frac{\partial^{2} h_{i \bar{i}}}{\partial z^{k} \partial \bar{z}^{\ell}}\right)+\sum_{i, q}\left(\frac{\partial h_{q \bar{\ell}}}{\partial \bar{z}^{i}} \frac{\partial h_{k \bar{q}}}{\partial z^{i}}+\frac{\partial h_{k \bar{q}}}{\partial \bar{z}^{i}} \frac{\partial h_{q \bar{\ell}}}{\partial z^{i}}\right) \\
& -2 \sum_{q, i}\left(\frac{\partial h_{q \bar{\ell}}}{\partial z^{k}} \frac{\partial h_{i \bar{q}}}{\partial \bar{z}^{i}}+\frac{\partial h_{q \bar{i}}}{\partial z^{i}} \frac{\partial h_{k \bar{q}}}{\partial \bar{z}^{\ell}}\right)
\end{aligned}
$$

Proposition 3.7. If $(M, \omega)$ is a compact Hermitian manifold with $\Lambda(\partial \bar{\partial} \omega)=0$, then

$$
B^{(2)} \leq \Theta^{(1)} \quad \text { and } \quad B^{(1)} \leq \Theta^{(2)}
$$


in the sense of Hermitian matrices and identities hold if and only if $(M, \omega)$ is Kähler. Moreover,

$$
\Theta^{(2)}+B^{(2)}=\Theta^{(1)}+R^{(1)}
$$

Finally, we would like to discuss the relations of balanced manifolds and strong Kähler manifolds with torsion. By [2], every Moishezon manifold is balanced, i.e. there exists a smooth Hermitian metric $\omega$ such that $d^{*} \omega=0$. On the other hand, by Demailly-Paun [10]( see also [27]), on each Moishezon manifold, there exists a singular Hermitian metric $\omega$ such that $\partial \bar{\partial} \omega=0$ in the sense of current. However, these two conditions can not be satisfied simultaneously in the smooth sense on an Hermitian non-Kähler manifold. It is known in [1] and also [15], but merits a proof in our setting.

Proposition 3.8. Let $(M, \omega)$ be a compact Hermitian manifold. If $d^{*} \omega=0$ and $\Lambda(\partial \bar{\partial} \omega)=0$, then $d \omega=0$, i.e. $(M, \omega)$ is Kähler. In particular, if a compact Hermitian manifold admits a smooth metric $\omega$ such that $d^{*} \omega=0$ and $\partial \bar{\partial} \omega=0$, then it is Kähler.

Proof. Let $(M, \omega)$ be a balanced Hermitian manifold with $\Lambda(\partial \bar{\partial} \omega)=0$. The condition $\Lambda(\partial \bar{\partial} \omega)=0$ is equivalent to

$$
\sum_{i} \frac{\partial h_{\bar{i} \bar{i}}}{\partial z^{k} \partial \bar{z}^{\ell}}+\sum_{i} \frac{\partial^{2} h_{k \bar{\ell}}}{\partial z^{i} \partial \bar{z}^{i}}=\sum_{i} \frac{\partial h_{i \bar{\ell}}}{\partial z^{k} \partial \bar{z}^{i}}+\sum_{i} \frac{\partial^{2} h_{k \bar{i}}}{\partial z^{i} \partial \bar{z}^{\ell}}
$$

By formula 3.5 , at a point $p$ with $h_{i \bar{j}}=\delta_{i j}$ and $\Gamma_{i j}^{k}(p)=0$, we have

$$
\begin{aligned}
\sum_{i} \frac{\partial h_{i \bar{i}}}{\partial z^{k} \partial \bar{z}^{\ell}}+\sum_{i} \frac{\partial^{2} h_{k \bar{\ell}}}{\partial z^{i} \partial \bar{z}^{i}} & =\sum_{i} \frac{\partial h_{i \bar{\ell}}}{\partial z^{k} \partial \bar{z}^{i}}+\sum_{i} \frac{\partial^{2} h_{k \bar{i}}}{\partial z^{i} \partial \bar{z}^{\ell}} \\
& =2 \sum_{i} \frac{\partial h_{\bar{i} \bar{i}}}{\partial z^{k} \partial \bar{z}^{\ell}}-4 \sum_{q, i} \frac{\partial h_{q \bar{\ell}}}{\partial \bar{z}^{i}} \frac{\partial h_{k \bar{q}}}{\partial z^{i}}
\end{aligned}
$$

That is

$$
\sum_{i} \frac{\partial h_{i \bar{i}}}{\partial z^{k} \partial \bar{z}^{\ell}}=\sum_{i} \frac{\partial^{2} h_{k \bar{\ell}}}{\partial z^{i} \partial \bar{z}^{i}}+4 \sum_{q, i} \frac{\partial h_{q \bar{\ell}}}{\partial \bar{z}^{i}} \frac{\partial h_{k \bar{q}}}{\partial z^{i}}
$$

Taking trace of it, we obtain

$$
4 \sum_{q, i, k} \frac{\partial h_{q \bar{k}}}{\partial \bar{z}^{i}} \frac{\partial h_{k \bar{q}}}{\partial z^{i}}=0 \Longleftrightarrow \frac{\partial h_{k \bar{q}}}{\partial z^{i}}=0
$$

at point $p$. Since $p$ is arbitrary, we have $d \omega \equiv 0$, therefore, $(M, \omega)$ is Kähler.

\section{Bochner formulas on Hermitian complex and Riemannian real vector bundles over compact Hermitian manifolds}

Let $(M, h, \omega)$ be a compact Hermitian manifold. The complexified Levi-Civita connection $\nabla$ on $T_{\mathbb{C}} M$ induces a linear connection on $\Omega^{p, q}(M)$ :

$$
\nabla: \Omega^{p, q}(M) \longrightarrow \Omega^{1}(M) \otimes\left(\Omega^{p, q}(M) \oplus \Omega^{p-1, q+1}(M) \oplus \Omega^{p+1, q-1}(M)\right)
$$

We consider the following two canonical components of $\nabla$,

$$
\left\{\begin{array}{l}
\nabla^{\prime}: \Omega^{p, q}(M) \rightarrow \Omega^{1,0}(M) \otimes \Omega^{p, q}(M) \\
\nabla^{\prime \prime}: \Omega^{p, q}(M) \rightarrow \Omega^{0,1}(M) \otimes \Omega^{p, q}(M)
\end{array}\right.
$$


Note that $\nabla \neq \nabla^{\prime}+\nabla^{\prime \prime}$ if $(M, h, \omega)$ is not Kähler. The following calculation rule follows immediately

$$
\nabla^{\prime}(\varphi \wedge \psi)=\left(\nabla^{\prime} \varphi\right) \wedge \psi+\varphi \wedge \nabla^{\prime} \psi
$$

for any $\varphi, \psi \in \Omega^{\bullet}(M)$.

Lemma 4.1. On an Hermitian manifold $(M, h)$, we have

$$
\left\{\begin{array} { l } 
{ \partial h ( \varphi , \psi ) = h ( \nabla ^ { \prime } \varphi , \psi ) + h ( \varphi , \nabla ^ { \prime \prime } \psi ) } \\
{ \overline { \partial } h ( \varphi , \psi ) = h ( \nabla ^ { \prime \prime } \varphi , \psi ) + h ( \varphi , \nabla ^ { \prime } \psi ) }
\end{array} \Longleftrightarrow \left\{\begin{array}{l}
\frac{\partial}{\partial z^{i}} h(\varphi, \psi)=h\left(\nabla_{i}^{\prime} \varphi, \psi\right)+h\left(\varphi, \nabla_{i}^{\prime \prime} \psi\right) \\
\frac{\partial}{\partial \bar{z}^{j}} h(\varphi, \psi)=h\left(\nabla_{\bar{j}}^{\prime \prime} \varphi, \psi\right)+h\left(\varphi, \nabla_{j}^{\prime} \psi\right)
\end{array}\right.\right.
$$

for any $\varphi, \psi \in \Omega^{p, q}(M)$.

Remark 4.2. (1) Here we use the compact notations

$$
\nabla_{i}^{\prime}=\nabla_{\frac{\partial}{\partial z^{i}}}^{\prime}, \quad \nabla_{j}^{\prime \prime}=\nabla_{\frac{\partial}{\partial \bar{z}^{j}}}^{\prime \prime}
$$

Note that $\nabla_{\bar{j}}^{\prime}=\nabla_{i}^{\prime \prime}=0$ and $\nabla_{i} \neq \nabla_{i}^{\prime}, \nabla_{\bar{j}} \neq \nabla_{\bar{j}}^{\prime}$.

(2) If we regard $\Lambda^{p, q} T^{*} M$ as an abstract vector bundle $E$, the above lemma says that $\nabla^{\prime}$ and $\nabla^{\prime \prime}$ are compatible with the Hermitian metric on $E$.

Now we go to an abstract setting. Let $E$ be an Hermitian complex (possibly non-holomorphic) vector bundle or a Riemannian real vector bundle over a compact Hermitian manifold $(M, \omega)$. There is a natural decomposition

$$
\nabla=\nabla^{\prime} E+\nabla^{\prime \prime E}
$$

where

$$
\left\{\begin{array}{l}
\nabla^{\prime} E: \Gamma(M, E) \longrightarrow \Omega^{1,0}(M, E) \\
\nabla^{\prime \prime} E: \Gamma(M, E) \longrightarrow \Omega^{0,1}(M, E)
\end{array}\right.
$$

$\nabla^{\prime} E$ and $\nabla^{\prime \prime} E$ induce two differential operators. The first one is $\partial_{E}: \Omega^{p, q}(M, E) \longrightarrow$ $\Omega^{p+1, q}(M, E)$ defined by

$$
\partial_{E}(\varphi \otimes s)=(\partial \varphi) \otimes s+(-1)^{p+q} \varphi \wedge \nabla^{\prime} s_{s}
$$

for any $\varphi \in \Omega^{p, q}(M)$ and $s \in \Gamma(M, E)$. The other one is $\bar{\partial}_{E}: \Omega^{p, q}(M, E) \longrightarrow \Omega^{p+1, q}(M, E)$ defined by

$$
\bar{\partial}_{E}(\varphi \otimes s)=(\bar{\partial} \varphi) \otimes s+(-1)^{p+q} \varphi \wedge \nabla^{\prime \prime} E_{s}
$$

for any $\varphi \in \Omega^{p, q}(M)$ and $s \in \Gamma(M, E)$. The following formula is well-known

$$
\left(\partial_{E} \bar{\partial}_{E}+\bar{\partial}_{E} \partial_{E}\right)(\varphi \otimes s)=\varphi \wedge\left(\partial_{E} \bar{\partial}_{E}+\bar{\partial}_{E} \partial_{E}\right) s
$$

for any $\varphi \in \Omega^{p, q}(M)$ and $s \in \Gamma(M, E)$. The operator $\partial_{E} \bar{\partial}_{E}+\bar{\partial}_{E} \partial_{E}$ is represented by its $(1,1)$ curvature tensor $R^{E} \in \Gamma\left(M, \Lambda^{1,1} T^{*} M \otimes E\right)$. For any $\varphi, \psi \in \Omega^{\bullet \bullet \bullet}(M, E)$, there is a sesquilinear pairing

$$
\{\varphi, \psi\}=\varphi^{\alpha} \wedge \overline{\psi^{\beta}}\left\langle e_{\alpha}, e_{\beta}\right\rangle
$$

if $\varphi=\varphi^{\alpha} e_{\alpha}$ and $\psi=\psi^{\beta} e_{\beta}$ in the local frames $\left\{e_{\alpha}\right\}$ on $E$. By the metric compatible property of $\nabla^{E}$,

$$
\partial\{\varphi, \psi\}=\left\{\partial_{E} \varphi, \psi\right\}+(-1)^{p+q}\left\{\varphi, \bar{\partial}_{E} \psi\right\}
$$

if $\varphi \in \Omega^{p, q}(M, E)$. 
Let $\omega$ be the Kähler form of the Hermitian metric $h$, i.e.,

$$
\omega=\frac{\sqrt{-1}}{2} h_{i \bar{j}} d z^{i} \wedge d \bar{z}^{j}
$$

On the Hermitian manifold $(M, h, \omega)$, the norm on $\Omega^{p, q}(M)$ is defined by

$$
(\varphi, \psi)=\int_{M}\langle\varphi, \psi\rangle \frac{\omega^{n}}{n !}=\frac{2^{n}}{(p+q) !} \int_{M} h(\varphi, \psi) \frac{\omega^{n}}{n !}=\int_{M} \varphi \wedge * \bar{\psi}
$$

The norm on $\Omega^{p, q}(M, E)$ is defined by

$$
(\varphi, \psi)=\int_{M}\{\varphi, * \psi\}=\int_{M}\left(\varphi^{\alpha} \wedge * \overline{\psi^{\beta}}\right)\left\langle e_{\alpha}, e_{\beta}\right\rangle
$$

for $\varphi, \psi \in \Omega^{p, q}(M, E)$. The dual operators of $\partial, \bar{\partial}, \partial_{E}$ and $\bar{\partial}_{E}^{*}$ are denoted by $\partial^{*}, \bar{\partial}^{*}, \partial_{E}^{*}$ and $\bar{\partial}_{E}^{*}$ respectively.

The following lemma was firstly shown by Demailly using Taylor expansion method( e.g. [8]). For the convenience of the reader, we will take another approach which seems to be useful in local computations.

Lemma 4.3. Let $(M, h, \omega)$ be a compact Hermitian manifold. If $\tau$ is the operator of type $(1,0)$ defined by $\tau=[\Lambda, 2 \partial \omega]$ on $\Omega^{\bullet}(M, E)$,

$$
\left\{\begin{array}{l}
{[\Lambda, \partial]=\sqrt{-1}\left(\bar{\partial}^{*}+\bar{\tau}^{*}\right)} \\
{[\Lambda, \bar{\partial}]=-\sqrt{-1}\left(\partial^{*}+\tau^{*}\right)}
\end{array}\right.
$$

For the dual equation, it is

$$
\left\{\begin{array}{l}
{\left[\bar{\partial}^{*}, L\right]=\sqrt{-1}(\partial+\tau)} \\
{\left[\partial^{*}, L\right]=-\sqrt{-1}(\bar{\partial}+\bar{\tau})}
\end{array}\right.
$$

where $L$ is the operator $L \varphi=2 \omega \wedge \varphi$ and $\Lambda$ is the adjoint operator of $L$.

Proof. See Appendix Lemma 8.7.

In the rest of this section $E$ is assumed to be an Hermitian complex vector bundles or a Riemannian real vector bundle over a compact Hermitian manifold $M$.

Lemma 4.4. Let $\nabla^{E}$ be a metric connection on $E$ over a compact Hermitian manifold $(M, \omega)$. If $\tau$ is the operator of type $(1,0)$ defined by $\tau=[\Lambda, 2 \partial \omega]$ on $\Omega^{\bullet}(M, E)$, then

(1) $\left[\bar{\partial}_{E}^{*}, L\right]=\sqrt{-1}\left(\partial_{E}+\tau\right)$;

(2) $\left[\partial_{E}^{*}, L\right]=-\sqrt{-1}\left(\bar{\partial}_{E}+\bar{\tau}\right)$;

(3) $\left[\Lambda, \partial_{E}\right]=\sqrt{-1}\left(\bar{\partial}_{E}^{*}+\bar{\tau}^{*}\right)$;

(4) $\left[\Lambda, \bar{\partial}_{E}\right]=-\sqrt{-1}\left(\partial_{E}^{*}+\tau^{*}\right)$.

Proof. See Appendix Lemma 8.10.

Theorem 4.5. Let $\nabla^{E}$ be a metric connection $E$ over a compact Hermitian manifold $(M, \omega)$.

$$
\Delta_{\bar{\partial}_{E}}=\Delta_{\partial_{E}}+\sqrt{-1}\left[\partial_{E} \bar{\partial}_{E}+\bar{\partial}_{E} \partial_{E}, \Lambda\right]+\left(\partial_{E} \tau^{*}+\tau^{*} \partial_{E}\right)-\left(\bar{\partial}_{E} \bar{\tau}^{*}+\bar{\tau}^{*} \bar{\partial}_{E}\right)
$$

where

$$
\left\{\begin{array}{l}
\Delta_{\bar{\partial}_{E}}=\bar{\partial}_{E} \bar{\partial}_{E}^{*}+\bar{\partial}_{E}^{*} \bar{\partial}_{E} \\
\Delta_{\partial_{E}}=\partial_{E} \partial_{E}^{*}+\partial_{E}^{*} \partial_{E}
\end{array}\right.
$$


Proof. It follows from Lemma 4.4.

We make a useful observation on the torsion $\tau$ :

Lemma 4.6. For any $s \in \Gamma(M, E)$, we have

$$
\tau(s)=-2 \sqrt{-1}\left(\bar{\partial}^{*} \omega\right) \cdot s, \quad \bar{\tau}(s)=2 \sqrt{-1}\left(\partial^{*} \omega\right) \cdot s
$$

Proof. By definition

$$
\begin{aligned}
([\Lambda, 2 \partial \omega]) s & =2 \Lambda((\partial \omega) \cdot s) \\
& =2(\Lambda(\partial \omega)) \cdot s \\
& =-2 \sqrt{-1}\left(\bar{\partial}^{*} \omega\right) \cdot s
\end{aligned}
$$

Here we use the identity

$$
\bar{\partial}^{*} \omega=\sqrt{-1} \Lambda(\partial \omega)
$$

where the proof of it is contained in Lemma 8.8 of the Appendix.

Corollary 4.7. If $(M, \omega)$ is a compact balanced Hermitian manifold, and $\nabla^{E}$ a metric connection on $E$ over $M$, then

$$
\left\|\bar{\partial}_{E} s\right\|^{2}=\left\|\partial_{E} s\right\|^{2}+\left(\sqrt{-1}\left[\partial_{E} \bar{\partial}_{E}+\bar{\partial}_{E} \partial_{E}, \Lambda\right] s, s\right)
$$

for any $s \in \Gamma(M, E)$.

Proof. Since for any $s \in \Gamma(M, E), \tau s=\bar{\tau} s=0$ and $\tau^{*} s=\bar{\tau}^{*} s=0$ on a balanced Hermitian manifold.

Theorem 4.8. Let $(M, \omega)$ be an Hermitian manifold with $\partial \bar{\partial} \omega^{n-1}=0$. If $\nabla^{E}$ is a metric connection on $E$ over $M$, then

$$
0=\left\|\bar{\partial}_{E} s\right\|^{2}=\left\|\partial_{E} s\right\|^{2}+\left(\sqrt{-1}\left[\partial_{E} \bar{\partial}_{E}+\bar{\partial}_{E} \partial_{E}, \Lambda\right] s, s\right)
$$

for any $s \in \Gamma(M, E)$ with $\bar{\partial}_{E} s=0$.

Proof. We only have to prove that

$$
\left(\left(\partial_{E} \tau^{*}+\tau^{*} \partial_{E}\right) s-\left(\bar{\partial}_{E} \bar{\tau}^{*}+\bar{\tau}^{*} \bar{\partial}_{E}\right) s, s\right)=0
$$

which is equivalent to $\left(\partial_{E} s, \tau s\right)=0$ since $\tau^{*} s=\bar{\tau}^{*} s=\bar{\partial}_{E} s=0$. By formula 4.19 and Stokes' Theorem,

$$
\begin{aligned}
\left(\tau^{*} \partial_{E} s, s\right) & =\left(\partial_{E} s, \tau s\right)=\int_{M}\left\{\partial_{E} s, *(\tau s)\right\} \\
& =2 \sqrt{-1} \int_{M}\left\{\partial_{E} s, *\left(\bar{\partial}^{*} \omega \cdot s\right)\right\} \\
& =2 \sqrt{-1} \int_{M}\left\{\partial_{E} s,\left(* \bar{\partial}^{*} \omega\right) \cdot s\right\} \\
& =-2 \sqrt{-1} \int_{M}\left\{s, \bar{\partial}_{E}\left(\left(* \bar{\partial}^{*} \omega\right) \cdot s\right)\right\} \\
& =-2 \sqrt{-1} \int_{M}\left\{s,\left(\bar{\partial}^{*} \bar{\partial}^{*} \omega\right) \cdot s-\left(* \bar{\partial}^{*} \omega\right) \wedge \bar{\partial}_{E} s\right\}
\end{aligned}
$$


It is easy to see that

$$
\bar{\partial} * \bar{\partial}^{*} \omega=-\bar{\partial} * * \partial * \omega=c_{n} \bar{\partial} \partial \omega^{n-1}=0
$$

since $* \omega=c_{n} \omega^{n-1}$ where $c_{n}$ is a constant depending only on the complex dimension of $M$. Hence

$$
\left(\partial_{E} s, \tau s\right)=2 \sqrt{-1} \int_{M}\left\{s,\left(* \bar{\partial}^{*} \omega\right) \wedge \bar{\partial}_{E} s\right\}=0
$$

since $\bar{\partial}_{E} s=0$.

Remark 4.9. By these formula, we can obtain classical vanishing theorems on Kähler manifolds and rigidity of harmonic maps between Hermitian and Riemannian manifolds.

\section{Vanishing theorems on Hermitian manifolds}

\subsection{Vanishing theorems on compact Hermitian manifolds}

Let $E$ be an Hermitian complex (possibly non-holomorphic) vector bundle or a Riemannian real vector bundle over a compact Hermitian manifold $(M, \omega)$. Let $\partial_{E}, \bar{\partial}_{E}$ be the $(1,0),(0,1)$ part of $\nabla^{E}$ respectively. The $(1,1)$-curvature of $\nabla^{E}$ is denoted by $R^{E} \in \Gamma\left(M, \Lambda^{1,1} T^{*} M \otimes\right.$ $\left.E^{*} \otimes E\right)$. It is a representation of the operator $\partial_{E} \bar{\partial}_{E}+\bar{\partial}_{E} \partial_{E}$. We can define harmonic section spaces associated to $\left(E, \nabla^{E}\right)$ by

$$
\mathcal{H}_{\bar{\partial}_{E}}^{p, q}(M, E)=\left\{\varphi \in \Omega^{p, q}(M, E) \mid \bar{\partial}_{E} \varphi=\bar{\partial}_{E}^{*} \varphi=0\right\}
$$

In general, on a complex vector bundle $E$, there is no such terminology like "holomorphic section of $E$ ". However, if the vector bundle $E$ is holomorphic and $\nabla^{E}$ is the Chern connection of $E$ i.e. $\bar{\partial}_{E}=\bar{\partial}$, then $\mathcal{H}_{\bar{\partial}_{E}}^{p, q}(M, E)$ is isomorphic to the Dolbeault cohomology group $H_{\bar{\partial}}^{p, q}(M, E)$ and $H_{\bar{\partial}}(M, E)$ is the holomorphic section spaces $H^{0}(M, E)$ of $E$.

Definition 5.1. Let $A$ be an $r \times r$ Hermitian matrix and $\lambda_{1} \leq \cdots \leq \lambda_{r}$ be eigenvalues of $A$. $A$ is said to be $p$-nonnegative (resp. positive, negative, nonpositive) for $1 \leq p \leq r$ if

$$
\lambda_{i_{1}}+\cdots+\lambda_{i_{p}} \geq 0(\text { resp. } \quad>0,<0, \leq 0) \text { for any } 1 \leq i_{1}<i_{2}<\cdots<i_{p} \leq n
$$

Theorem 5.2. Let $\nabla^{E}$ be any metric connection of an Hermitian complex vector bundle or a Riemannian real vector bundle $E$ over a compact Hermitian manifold $(M, h, \omega)$.

(1) If the second Hermitian-Ricci curvature $\operatorname{Tr}_{\omega} R^{E}$ is nonpositive everywhere, then every $\bar{\partial}_{E}$-closed section of $E$ is parallel, i.e. $\nabla^{E} s=0$;

(2) If the second Hermitian-Ricci curvature $\operatorname{Tr}_{\omega} R^{E}$ is nonpositive everywhere and negative at some point, then $\mathcal{H}_{\partial_{E}}^{0}(M, E)=0$;

(3) If the second Hermitian-Ricci curvature $\operatorname{Tr}_{\omega} R^{E}$ is p-nonpositive everywhere and $p$-negative at some point, then $\mathcal{H}_{\bar{\partial}_{E}}^{0}\left(M, \Lambda^{q} E\right)=0$ for any $p \leq q \leq \operatorname{rank}(E)$.

Proof. By [21], there exists a smooth function $u: M \longrightarrow \mathbb{R}$ such that $\omega_{G}=e^{u} \omega$ is a Gauduchon metric, i.e. $\partial \bar{\partial} \omega_{G}^{n-1}=0$. Now we replace the metric $\omega$ on $M$ by the Gauduchon metric $\omega_{G}$. By the relation $\omega_{G}=e^{u} \omega$, we get

$$
\operatorname{Tr}_{\omega_{G}} R^{E}=e^{-u} \operatorname{Tr}_{\omega} R^{E}
$$


Therefore, the positivity conditions in the Theorem are preserved. Let $s \in \Gamma(M, E)$ with $\bar{\partial}_{E} s=0$, by formula 4.22 , we obtain

$$
0=\left\|\partial_{E} s\right\|^{2}+\left(\sqrt{-1}\left[\partial_{E} \bar{\partial}_{E}+\bar{\partial}_{E} \partial_{E}, \Lambda_{G}\right] s, s\right)=\left\|\partial_{E} s\right\|^{2}-\left(T r_{\omega_{G}} R^{E} s, s\right)
$$

where

$$
R^{E}=\partial_{E} \bar{\partial}_{E}+\bar{\partial}_{E} \partial_{E}=R_{i \bar{j} \alpha}^{\beta} d z^{i} \wedge d \bar{z}^{j} \otimes e^{\alpha} \otimes e_{\beta}
$$

Since the second Hermitian-Ricci curvature $\operatorname{Tr}_{\omega_{G}} R^{E}$ has components

$$
R_{\alpha \bar{\beta}}=h_{G}^{i \bar{j}} R_{i \bar{j} \alpha \bar{\beta}}
$$

formula 5.4 can be written as

$$
0=\left\|\partial_{E} s\right\|^{2}-\int_{M} R_{\alpha \bar{\beta}} s^{\alpha} \bar{s}^{\beta}
$$

Now (1) and (2) follow by identity 5.7 with the curvature conditions immediately. For (3), we set $F=\Lambda^{q} E$ with $p \leq q \leq r=\operatorname{rank}(E)$. Let $\lambda_{1} \leq \cdots \leq \lambda_{r}$ be the eigenvalues of $\operatorname{Tr}_{\omega_{G}} R^{E}$, then we know

$$
\lambda_{1}+\cdots+\lambda_{p} \geq 0
$$

and it is strictly positive at some point. If $p \leq q \leq r$, the smallest eigenvalue of $\operatorname{Tr}_{\omega_{G}} R^{F}$ is $\lambda_{1}+\cdots+\lambda_{q} \geq 0$ and it is strictly positive at some point. By (2), we know $\mathcal{H}_{\bar{\partial}_{E}}^{0}(M, F)=0$.

If $\nabla^{E}$ is the Chern connection of the Hermitian holomorphic vector bundle $E$, we know

$$
\mathcal{H}_{\bar{\partial}_{E}}^{0}(M, E) \cong H^{0}(M, E)
$$

since $\bar{\partial}_{E}=\nabla^{\prime \prime} E=\bar{\partial}$ for the Chern connection.

Corollary 5.3 (Kobayashi-Wu[31], Gauduchon [19]). Let $\nabla^{E}$ be the Chern connection of an Hermitian holomorphic vector bundle $E$ over a compact Hermitian manifold $(M, h, \omega)$.

(1) If the second Ricci-Chern curvature $\operatorname{Tr}_{\omega} R^{E}$ is nonpositive everywhere, then every holomorphic section of $E$ is parallel, i.e. $\nabla^{E} s=0$;

(2) If the second Ricci-Chern curvature $\operatorname{Tr}_{\omega} R^{E}$ is nonpositive everywhere and negative at some point, then $E$ has no holomorphic section, i.e. $H^{0}(M, E)=0$;

(3) If the second Ricci-Chern curvature $\operatorname{Tr}_{\omega} R^{E}$ is p-nonpositive everywhere and $p$-negative at some point, then $\Lambda^{q} E$ has no holomorphic section for any $p \leq p \leq \operatorname{rank}(E)$.

Now we can apply it to the tangent and cotangent bundles of compact Hermitian manifolds.

Corollary 5.4. Let $(M, \omega)$ be a compact Hermitian manifold and $\Theta$ is the Chern curvature of the Chern connection $\nabla^{C H}$ on the holomorphic tangent bundle $T^{1,0} M$.

(1) If the second Ricci-Chern curvature $\Theta^{(2)}$ is nonpositive everywhere and negative at some point, then $M$ has no holomorphic vector field, i.e. $H^{0}\left(M, T^{1,0} M\right)=0$;

(2) If the second Ricci-Chern curvature $\Theta^{(2)}$ is nonnegative everywhere and positive at some point, then $M$ has no holomorphic $p$-form for any $1 \leq p \leq n$, i.e. $H_{\bar{\partial}}^{p, 0}(M)=0$; In particular, the arithmetic genus

$$
\chi(M, \mathcal{O})=\sum(-1)^{p} h^{p, 0}(M)=1
$$


(3) If the second Ricci-Chern curvature $\Theta^{(2)}$ is p-nonnegative everywhere and p-positive at some point, then $M$ has no holomorphic $q$-form for any $p \leq q \leq n$, i.e. $H_{\bar{\partial}}^{q, 0}(M)=0$. In particular, if the scalar curvature $S^{C H}$ is nonnegative everywhere and positive at some point, then $H^{0}\left(M, m K_{M}\right)=0$ for all $m \geq 1$ where $K_{M}$ is the canonical line bundle of $M$.

Proof. Let $E=T^{1,0} M$ and $h$ be an Hermitian metric on $E$ such that the second Ricci-Chern curvature $\operatorname{Tr}_{\omega_{h}} \Theta$ of $(E, h)$ satisfies the assumption. It is obvious that all section spaces in consideration are independent of the choice of the metrics and connections.

The metric on the vector bundle $E$ is fixed. Now we choose a Gauduchon metric $\omega_{G}=e^{u} \omega_{h}$ on $M$. Then the second Ricci-Chern curvature $\widetilde{\Theta}^{(2)}=\operatorname{Tr}_{\omega_{G}} \Theta=e^{-u} \operatorname{Tr}_{\omega_{h}} \Theta$ shares the semidefinite property with $\Theta^{(2)}=\operatorname{Tr}_{\omega_{h}} \Theta$. For the safety, we repeat the arguments in Theorem 5.2 briefly. If $s$ is a holomorphic section of $E$, i.e., $\bar{\partial}_{E} s=\bar{\partial} s=0$, by formula 4.22 , we obtain

$$
0=\left\|\partial_{E} s\right\|^{2}+\left(\sqrt{-1}\left[\partial_{E} \bar{\partial}_{E}+\bar{\partial}_{E} \partial_{E}, \Lambda_{G}\right] s, s\right)=\left\|\partial_{E} s\right\|^{2}-\left(\operatorname{Tr}_{\omega_{G}} \Theta s, s\right)
$$

If $\operatorname{Tr}_{\omega} \Theta$ is nonpositive everywhere, then $\partial_{E} s=0$ and so $\nabla^{E} s=0$. If $\operatorname{Tr}_{\omega} \Theta$ is nonpositive everywhere and negative at some point, we get $s=0$, therefore $H^{0}\left(M, T^{1,0} M\right)=0$. The proofs of the other parts are similar.

Remark 5.5. It is well-known that the first Ricci-Chern curvature $\Theta^{(1)}$ represents the first Chern class of $M$. But on an Hermitian manifold, it is possible that the second Ricci-Chern curvature $\Theta^{(2)}$ is not in the same $(d, \partial, \bar{\partial})$-cohomology class as $\Theta^{(1)}$. For example, $\mathbb{S}^{3} \times \mathbb{S}^{1}$ with canonical metric has strictly positive second Ricci-Chern curvature but it is well-known that it has vanishing first Chern number $c_{1}^{2}$. For more details see Proposition 6.4. Therefore, $\Theta^{(2)}$ in Proposition 5.4 can NOT be replaced by $\Theta^{(1)}$. It seems to be an interesting question: if $(M, \omega)$ is a compact Hermitian manifold and its first Ricci-Chern curvature is nonnegative everywhere and positive at some point, is the first Betti number of $M$ zero? In particular, is it Kähler in dimension 2?

As special cases of our results, the following results for Kähler manifolds are well-known, and we list them here for the convenience of the reader. Let $(M, h, \omega)$ be a compact Kähler manifold.

(1) If the Ricci curvature is nonnegative everywhere, then any holomorphic $(p, 0)$ form is parallel;

(2) If the Ricci curvature is nonnegative everywhere and positive at some point, then $h^{p, 0}=0$ for $p=1, \cdots, n$. In particular, the arithmetic genus $\chi(M, \mathcal{O})=1$ and $b_{1}(M)=0$;

(3) If the scalar curvature is nonnegative everywhere and positive at some point, then $h^{n, 0}=$ 0 .

(A) If the Ricci curvature is nonpositive everywhere, then any holomorphic vector field is parallel;

(B) If the Ricci curvature is nonpositive everywhere and negative at some point, there is no holomorphic vector field. 


\subsection{Vanishing theorems on special Hermitian manifolds}

Let $(M, h, \omega)$ be a compact Hermitian manifold and $\nabla$ be the Levi-Civita connection.

Lemma 5.6. Let $(M, \omega)$ be a compact balanced Hermitian manifold. For any $(p, 0)$-form $\varphi$ on $M$,

(1) If $\varphi$ is holomorphic, then $\partial^{*} \varphi=0$;

(2) If $\nabla^{\prime} \varphi=0$, then $\partial \varphi=0$.

Proof. For simplicity, we assume $p=1$. For the general case, the proof is the same. By Lemma 8.5, we know, for any $(1,0)$-form $\varphi=\varphi_{i} d z^{i}$,

$$
\partial^{*} \varphi=-h^{i \bar{j}} \frac{\partial \varphi_{i}}{\partial \bar{z}^{j}}
$$

where we use the balanced condition $h^{i \bar{j}} \Gamma_{i \bar{j}}^{s}=0$. If $\varphi$ is holomorphic, then $\frac{\partial \varphi_{i}}{\partial \bar{z}^{j}}=0$, hence $\partial^{*} \varphi=0$. On the other hand,

$$
\nabla^{\prime} \varphi=\left(\frac{\partial \varphi_{i}}{\partial z^{j}}-\Gamma_{j i}^{m} \varphi_{m}\right) d z^{j} \otimes d z^{i}
$$

If $\nabla^{\prime} \varphi=0$, we obtain

$$
\partial \varphi=\frac{\partial \varphi_{i}}{\partial z^{j}} d z^{j} \wedge d z^{i}=\Gamma_{j i}^{m} \varphi_{m} d z^{j} \wedge d z^{i}=0
$$

Theorem 5.7. Let $(M, \omega)$ be a compact balanced Hermitian manifold with Levi-Civita connection $\nabla$.

(1) If the Hermitian-Ricci curvature $\left(R_{i \bar{j}}\right)$ is p-nonnegative everywhere, then any holomorphic $(q, 0)$-form $(p \leq q \leq n)$ is $\partial$-harmonic; in particular, $h^{q, 0}(M) \leq h^{0, q}(M)$ for any $p \leq q \leq n ;$

(2) If the Hermitian-Ricci curvature $\left(R_{i \bar{j}}\right)$ is p-nonnegative everywhere and p-positive at some point, $H_{\bar{\partial}}^{q, 0}(M)=0$ for any $p \leq q \leq n$;

In particular,

(3) if the Hermitian-Ricci curvature $\left(R_{i \bar{j}}\right)$ is nonnegative everywhere and positive at some point, then $H_{\bar{\partial}}^{p, 0}(M)=0$, for $p=1, \cdots, n$ and so the arithmetic genus $\chi(M, \mathcal{O})=1$ and $b_{1}(M) \leq h^{0,1}(M)$.

(4) if the Hermitian-scalar curvature $S$ is nonnegative everywhere and positive at some point, then

$$
H^{0}\left(M, m K_{M}\right)=0 \quad \text { for any } \quad m \geq 1
$$

where $K_{M}=\operatorname{det} T^{* 1,0} M$.

Proof. At first, we assume $p=1$ for (1) and (2). Now we consider $E=T^{* 1,0} M$ with the induced metric connection $\nabla^{E}=\widehat{\nabla}$ for $h$ (see 2.22). By formula 4.7, we have

$$
\left\|\bar{\partial}_{E} s\right\|^{2}=\left\|\partial_{E} s\right\|^{2}+\sqrt{-1}\left(\left[R^{E}, \Lambda\right] s, s\right)
$$


where $R^{E}$ is the $(1,1)$-part curvature of $E$ with respect to the connection $\nabla^{E}$. More precisely,

$$
R^{E}=\partial_{E} \bar{\partial}_{E}+\bar{\partial}_{E} \partial_{E}=-\widehat{R}_{i \bar{j} k}^{\ell} d z^{i} \wedge d \bar{z}^{j} \otimes \frac{\partial}{\partial z^{\ell}} \otimes d z^{k}
$$

since $E$ is the dual vector bundle of $T^{1,0} M$ and the $(1,1)$-part of the curvature of $T^{1,0} M$ is

$$
\widehat{R}_{i \bar{j} k}^{\ell} d z^{i} \wedge d \bar{z}^{j} \otimes d z^{k} \otimes \frac{\partial}{\partial z^{\ell}}
$$

If $s=f_{i} d z^{i}$ is a holomorphic 1-form, i.e.

$$
\bar{\partial} s=\frac{\partial f_{i}}{\partial \bar{z}^{j}} d \bar{z}^{j} \wedge d z^{i}=0
$$

then

$$
\bar{\partial}_{E} s=\left(\frac{\partial f_{i}}{\partial \bar{z}^{j}}-f_{k} \Gamma \frac{k}{j i}\right) d \bar{z}^{j} \otimes d z^{i}=-f_{k} \Gamma_{\bar{j} i}^{k} d \bar{z}^{j} \otimes d z^{i}
$$

Without loss of generality, we assume $h_{i \bar{j}}=\delta_{i j}$ at a given point. By Proposition 2.12, the quantity

$$
\left|\bar{\partial}_{E} s\right|^{2}=\sum_{i, j, t, n} f_{i} \bar{f}_{n} \Gamma_{\bar{j} t \bar{i}} \overline{\Gamma_{\bar{j} t \bar{n}}}=\sum_{i, n}\left(\widehat{R}_{n \bar{i}}^{(2)}-R_{n \bar{i}}\right) f_{i} \bar{f}_{n}
$$

On the other hand

$$
\sqrt{-1}\left\langle\left[R^{E}, \Lambda\right] s, s\right\rangle=\sum_{i, n} \widehat{R}_{n \bar{i}}^{(2)} f_{i} \bar{f}_{n}
$$

That is

$$
\left|\bar{\partial}_{E} s\right|^{2}-\sqrt{-1}\left\langle\left[R^{E}, \Lambda\right] s, s\right\rangle=-\sum_{i, n} R_{n \bar{i}} f_{i} \bar{f}_{n} \leq 0
$$

if the Hermitian-Ricci curvature $\left(R_{n \bar{i}}\right)$ of $(M, h, \omega)$ is nonnegative everywhere. Then we get

$$
0 \leq\left\|\partial_{E} s\right\|^{2}=\left\|\bar{\partial}_{E} s\right\|^{2}-\sqrt{-1}\left(\left[R^{E}, \Lambda\right] s, s\right) \leq 0
$$

That is $\partial_{E} s=0$. Since

$$
\partial_{E} s=\nabla^{\prime} E s=\widehat{\nabla}^{\prime} s=\nabla^{\prime} s=\left(\frac{\partial f_{i}}{\partial z^{j}}-f_{\ell} \Gamma_{i j}^{\ell}\right) d z^{j} \otimes d z^{i}
$$

we obtain $\nabla^{\prime} s=0$. By Lemma 5.6, we know $\Delta_{\partial} s=0$. In summary, we get

$$
H_{\bar{\partial}}^{1,0}(M) \subset H_{\partial}^{1,0}(M) \cong H_{\bar{\partial}}^{0,1}(M)
$$

If the Hermitian-Ricci curvature $\left(R_{n \bar{i}}\right)$ is nonnegative everywhere and positive at some point, then $f_{i}=0$ for each $i$, that is $s=0$. So we proved $H_{\bar{\partial}}^{1,0}(M)=0$. The general cases follow by the same arguments as Theorem 5.2 and Theorem 5.4.

The dual of Theorem 5.7 is

Theorem 5.8. Let $(M, h, \omega)$ be a compact balanced Hermitian manifold.

(1) If $2 \widehat{R}_{i \bar{j}}^{(2)}-R_{i \bar{j}}$ is nonpositive everywhere, then any holomorphic vector field is $\nabla^{\prime}$-closed;

(2) If $2 \widehat{R}_{i \bar{j}}^{(2)}-R_{i \bar{j}}$ is nonpositive everywhere and negative at some point, there is no holomorphic vector field. 
Proof. Let $E=T^{1,0} M$ and $\widehat{\nabla}$ the induced connection on it. If $s=f^{i} \frac{\partial}{\partial z^{i}}$ is a holomorphic section, then

$$
\bar{\partial}_{E} s=f^{i} \Gamma_{\bar{j} i}^{\ell} d \bar{z}^{j} \otimes \frac{\partial}{\partial z^{\ell}}
$$

Without loss generality, we assume $h_{i \bar{j}}=\delta_{i j}$ at a given point. By Proposition 2.12,

$$
\begin{aligned}
\left|\bar{\partial}_{E} s\right|^{2}-\sqrt{-1}\left\langle\left[\widehat{R}^{1,1}, \Lambda\right] s, s\right\rangle & =\left(\widehat{R}_{i \bar{j}}^{(2)}-R_{i \bar{j}}\right) f^{i} \bar{f}^{j}+\widehat{R}_{i \bar{j}}^{(2)} f^{i} \bar{f}^{j} \\
& =\left(2 \widehat{R}_{i \bar{j}}^{(2)}-R_{i \bar{j}}\right) f^{i} \bar{f}^{j}
\end{aligned}
$$

By formula 4.17,

$$
0 \leq\left\|\partial_{E} s\right\|^{2}=\left\|\bar{\partial}_{E} s\right\|^{2}-\sqrt{-1}\left(\left[\widehat{R}^{1,1}, \Lambda\right] s, s\right)
$$

So if $2 \widehat{R}_{i \bar{j}}^{(2)}-R_{i \bar{j}}$ is nonpositive everywhere, $\partial_{E} s=\nabla^{\prime} s=0$. If $2 \widehat{R}_{i \bar{j}}^{(2)}-R_{i \bar{j}}$ is nonpositive everywhere and negative at some point, there is no holomorphic vector field.

Remark 5.9. (1) It is obvious that the second Ricci-Chern curvature $\Theta_{k \bar{\ell}}^{(2)}$ and HermitianRicci curvature $R_{k \bar{\ell}}$ can not be compared. Therefore, Theorem 5.4 and Theorem 5.7 are independent of each other. For the same reason, Theorem 5.4 and Theorem 5.8 are independent.

(2) For a special case in Theorem 5.7, if the Hermitian-Ricci curvature $R_{k \bar{\ell}}$ is nonnegative everywhere and positive at some point, by Proposition 3.5, the manifold $(M, \omega)$ is Moishezon. It is well-known that every 2-dimensional Moishezon/balanced manifold is Kähler, but there are many Moishezon non-Kähler manifolds in higher dimension( See [36]).

The following result was firstly obtained in [25]:

Corollary 5.10. Let $(M, \omega)$ be a compact Hermitian manifold with $\Lambda(\partial \bar{\partial} \omega)=0$. Let $\nabla^{B}$ be the Bismut connection on $T^{1,0} M$.

(1) If the first Ricci-Bismut curvature $B^{(1)}$ is nonnegative everywhere, then every holomorphic $(p, 0)$-form is parallel with respect to the Chern connection $\nabla^{C H}$;

(2) If the first Ricci-Bismut curvature $B^{(1)}$ is nonnegative everywhere and positive at some point, then $M$ has no holomorphic $(p, 0)$-form for any $1 \leq p \leq n$, i.e. $H_{\bar{\partial}}^{p, 0}(M)=0$; in particular, the arithmetic genus $\chi(M, \mathcal{O})=1$.

(3) If the first Ricci-Bismut curvature $B^{(1)}$ is p-nonnegative everywhere and p-positive at some point then $M$ has no holomorphic $(q, 0)$-form for any $p \leq q \leq n$, i.e. $H_{\frac{q, 0}{\partial}}(M)=0$. In particular, if the scalar curvature $S^{B M}$ of the Bismut connection is nonnegative everywhere and positive at some point, then $H^{0}\left(M, m K_{M}\right)=0$ for any $m \geq 1$.

Proof. By Proposition 3.7, if $\Lambda(\partial \bar{\partial} \omega)=0$, then

$$
B^{(1)} \leq \Theta^{(2)}
$$

Now we can apply Corollary 5.4 to get (1), (2) and (3).

Remark 5.11. For more vanishing theorems on special Hermitian manifolds, one can see [1], [25], [16], [17] and references therein. 


\section{Examples of non-Kähler manifolds with nonnegative curva- tures}

Let $M=\mathbb{S}^{2 n-1} \times \mathbb{S}^{1}$ be the standard $n$-dimensional $(n \geq 2)$ Hopf manifold. It is diffeomorphic to $\mathbb{C}^{n}-\{0\} / G$ where $G$ is cyclic group generated by the transformation $z \rightarrow \frac{1}{2} z$. It has an induced complex structure of $\mathbb{C}^{n}-\{0\}$. For more details about such manifolds, we refer the reader to [30]. On $M$, there is a natural metric

$$
h=\sum_{i=1}^{n} \frac{4}{|z|^{2}} d z^{i} \otimes d \bar{z}^{i}
$$

The following identities follow immediately

$$
\frac{\partial h_{k \bar{\ell}}}{\partial z^{i}}=-\frac{4 \delta_{k \ell} \bar{z}^{i}}{|z|^{4}}, \quad \frac{\partial h_{k \bar{\ell}}}{\partial \bar{z}^{j}}=-\frac{4 \delta_{k \ell} z^{j}}{|z|^{4}}
$$

and

$$
\frac{\partial^{2} h_{k \bar{\ell}}}{\partial z^{i} \partial \bar{z}^{j}}=-4 \delta_{k \ell} \frac{\delta_{i \bar{j}}|z|^{2}-2 \bar{z}^{i} z^{j}}{|z|^{6}}
$$

Example 6.1 (Curvatures of Chern connection). Direct computation shows that, the Chen curvature components are

$$
\Theta_{i \bar{j} k \bar{\ell}}=-\frac{\partial^{2} h_{k \bar{\ell}}}{\partial z^{i} \partial \bar{z}^{j}}+h^{p \bar{q}} \frac{\partial h_{k \bar{q}}}{\partial z^{i}} \frac{\partial h_{p \bar{\ell}}}{\partial \bar{z}^{j}}=\frac{4 \delta_{k l}\left(\delta_{i j}|z|^{2}-z^{j} \bar{z}^{i}\right)}{|z|^{6}}
$$

and the first and second Ricci-Chern curvatures are

$$
\Theta_{k \bar{\ell}}^{(1)}=\frac{n\left(\delta_{k \ell}|z|^{2}-z^{\ell} \bar{z}^{k}\right)}{|z|^{4}}, \quad \Theta_{k \bar{\ell}}^{(2)}=\frac{(n-1) \delta_{k \ell}}{|z|^{2}}
$$

It is easy to see that the eigenvalues of $\Theta^{(1)}$ are

$$
\lambda_{1}=0, \lambda_{2}=\cdots=\lambda_{n}=\frac{n}{|z|^{2}}
$$

Hence, $\Theta^{(1)}$ is nonnegative and 2-positive everywhere.

Example 6.2 (Curvatures of Levi-Civita connection). Similarly, we have

$$
\Gamma_{i k}^{\ell}=-\frac{\delta_{i \ell} \bar{z}^{k}+\delta_{k \ell} \bar{z}^{i}}{2|z|^{2}}, \quad \Gamma_{\bar{j} k}^{\ell}=\frac{\delta_{j k} z^{\ell}-\delta_{k \ell} z^{j}}{2|z|^{2}}
$$

and

$$
\begin{gathered}
\frac{\partial \Gamma_{i k}^{\ell}}{\partial \bar{z}^{j}}=-\frac{\delta_{k \ell} \delta_{i j}+\delta_{i \ell} \delta_{j k}}{2|z|^{2}}+\frac{\delta_{i \ell} z^{j} \bar{z}^{k}+\delta_{k \ell} z^{j} \bar{z}^{i}}{2|z|^{4}} \\
\frac{\partial \Gamma_{j k}^{\ell}}{\partial z^{i}}=\frac{\delta_{j k} \delta_{i \ell}-\delta_{k \ell} \delta_{i j}}{2|z|^{2}}-\frac{\left(\delta_{j k} z^{\ell}-\delta_{k \ell} z^{j}\right) \bar{z}^{i}}{2|z|^{4}}
\end{gathered}
$$

The complexified Riemannian curvature components are

$$
R_{i \bar{j} k}^{\ell}=-\left(\frac{\partial \Gamma_{i k}^{\ell}}{\partial \bar{z}^{j}}-\frac{\partial \Gamma_{\bar{j} k}^{\ell}}{\partial z^{i}}+\Gamma_{i k}^{s} \Gamma_{\bar{j} s}^{\ell}-\Gamma_{\bar{j} k}^{s} \Gamma_{i s}^{\ell}-\Gamma_{\bar{j} k}^{\bar{s}} \Gamma_{i \bar{s}}^{\ell}\right)=\frac{\delta_{i \ell} \delta_{j k}}{2|z|^{2}}-\frac{\delta_{i \ell} z^{j} \bar{z}^{k}+\delta_{j k} z^{\ell} \bar{z}^{i}}{4|z|^{4}}
$$

and

$$
R_{i \bar{j} k \bar{\ell}}=\frac{2 \delta_{i \ell} \delta_{j k}}{|z|^{4}}-\frac{\delta_{i \ell} z^{j} \bar{z}^{k}+\delta_{j k} z^{\ell} \bar{z}^{i}}{|z|^{6}}, \quad R_{k \bar{\ell}}=\frac{\delta_{k \ell}|z|^{2}-z^{\ell} \bar{z}^{k}}{2|z|^{4}}
$$


Example 6.3 ( Curvatures of Bismut connection). By definition 2.45 and Lemma 2.10, we obtain

$$
B_{i \bar{j} k}^{\ell}=\frac{\delta_{j k} \delta_{i \ell}-\delta_{k \ell} \delta_{i j}}{|z|^{2}}+\frac{\delta_{i j} \bar{z}^{k} z^{\ell}+\delta_{k \ell} \bar{z}^{i} z^{j}-\delta_{i \ell} \bar{z}^{k} z^{j}-\delta_{j k} \bar{z}^{i} z^{\ell}}{|z|^{4}}
$$

Two Ricci curvatures are

$$
B_{i \bar{j}}^{(1)}=B_{i \bar{j}}^{(2)}=\frac{(2-n)\left(\delta_{i j}|z|^{2}-\bar{z}^{i} z^{j}\right)}{4|z|^{2}}
$$

On the other hand, by 6.3 , it is easy to see $\partial \bar{\partial} \omega=0$ and $B^{(1)}=0$ for $n=2$.

Proposition 6.4. Let $M=\mathbb{S}^{2 n-1} \times \mathbb{S}^{1}$ be the standard $n$-dimensional $(n \geq 2)$ Hopf manifold with canonical metric $h$,

(1) $(M, h)$ has positive second Ricci-Chern curvature $\Theta^{(2)}$;

(2) $(M, h)$ has nonnegative first Ricci-Chern curvature $\Theta^{(1)}$, i.e., $c_{1}(M) \geq 0$. Moreover,

$$
\int_{M} c_{1}^{n}(M)=0
$$

(3) $(M, h)$ is semi-positive in the sense of Griffiths, i.e.

$$
\Theta_{i \bar{j} k \bar{\ell}} u^{i} \bar{u}^{j} v^{k} \bar{v}^{\ell} \geq 0
$$

for any $u, v \in \mathbb{C}^{n}$

(4) $R_{k \bar{\ell}}$ is nonnegative and 2-positive everywhere;

(5) $(M, h)$ has nonpositive and 2-negative first Ricci-Bismut curvature. In particular, $\left(\mathbb{S}^{3} \times\right.$ $\left.\mathbb{S}^{1}, \omega\right)$ satisfies $\partial \bar{\partial} \omega=0$ and has vanishing first Ricci-Bismut curvature $B^{(1)}$.

Although we know all Betti numbers of Hopf manifold $\mathbb{S}^{2 n-1} \times \mathbb{S}^{1}, h^{p, 0}$ is not so obvious.

Corollary 6.5. Let $(M, h)$ be $n$-dimensional Hopf manifold with $n \geq 2$,

(1) $h^{p, 0}(M)=0$ for $p \geq 1$ and $\chi(M, \mathcal{O})=1$. In particular, $h^{0,1}(M) \geq 1$.

(2) $\operatorname{dim}_{\mathbb{C}} H^{0}(M, m K)=0$ for any $m \geq 1$ where $K=\operatorname{det}\left(T^{* 1,0} M\right)$.

\section{A natural geometric flow on Hermitian manifolds}

As we discussed in the above sections, on Hermitian manifolds, the second Ricci curvature tensors of various metric connections are closely related to the geometry of Hermitian manifolds. A natural idea is to define a flow by using second Ricci curvature tensors of various metric connections. We describe it in the following.

Let $(M, h)$ be a compact Hermitian manifold. Let $\nabla$ be an arbitrary metric connection on the holomorphic tangent bundle $(E, h)=\left(T^{1,0} M, h\right)$.

$$
\nabla: E \longrightarrow \Omega^{1}(E)
$$

It has two components $\nabla^{\prime}$ and $\nabla^{\prime \prime}$,

$$
\nabla=\nabla^{\prime}+\nabla^{\prime \prime}
$$


$\nabla^{\prime}$ and $\nabla^{\prime \prime}$ induce two differential operators

$$
\begin{aligned}
& \partial_{E}: \Omega^{p, q}(E) \longrightarrow \Omega^{p+1, q}(E) \\
& \bar{\partial}_{E}: \Omega^{p, q}(E) \longrightarrow \Omega^{p, q+1}(E)
\end{aligned}
$$

Let $R^{E}$ be the $(1,1)$ curvature of the metric connection $\nabla$. More precisely $R^{E}$ is a representation of $\partial_{E} \bar{\partial}_{E}+\bar{\partial}_{E} \partial_{E}$. It is easy to see that

$$
R^{E} \in \Gamma\left(M, \Lambda^{1,1} T^{*} M \otimes \operatorname{End}(E)\right)
$$

and locally, we can write it as

$$
R^{E}=R_{i \bar{j} A}^{B} d z^{i} \wedge d z^{j} \otimes e^{A} \otimes e_{B}
$$

Here we set $e_{A}=\frac{\partial}{\partial z^{A}}, e^{B}=d z^{B}$ where $A, B=1, \cdots, n$, since the geometric meanings of $j$ and $A$ are different. It is well-known that a metric connection $\nabla$ is determined by its Christoffel symbols

$$
\nabla_{\frac{\partial}{\partial z^{i}}} e_{A}=\Gamma_{i A}^{B} e_{B}, \quad \nabla_{\frac{\partial}{\partial \bar{z}^{j}}} e_{A}=\Gamma_{j A}^{B} e_{B}
$$

In particular, we don't have notations such as $\Gamma_{A i}^{B}$. It is obvious that

$$
R_{i \bar{j} B}^{A}=-\frac{\partial \Gamma_{i A}^{B}}{\partial \bar{z}^{j}}+\frac{\partial \Gamma_{j}^{B} A}{\partial z^{i}}-\Gamma_{i A}^{C} \Gamma_{\bar{j} C}^{B}+\Gamma_{j A}^{C} \Gamma_{i C}^{B}
$$

We set the second Hermitian-Ricci curvature tensor of $(\nabla, h)$ as

$$
R^{(2)}=h^{i \bar{j}} R_{i \bar{j} A \bar{B}} e^{A} \otimes \bar{e}^{B} \in \Gamma\left(M, E^{*} \otimes \bar{E}^{*}\right)
$$

In general we can study a new class of flows on Hermitian manifolds

$$
\left\{\begin{array}{l}
\frac{\partial h}{\partial t}=\mathcal{F}(h)+\mu h \\
h(0)=h_{0}
\end{array}\right.
$$

where $\mathcal{F}$ can be a linear combination of the first and the second Hermitian-Ricci curvature tensors of different metric connections on $\left(T^{1,0} M, h\right)$. For examples, $\mathcal{F}(h)=-\Theta^{(2)}$, the second Ricci-Chern curvature tensor of the Chern connection, and $\mathcal{F}(h)=-\widehat{R}^{(2)}$, the second Hermitian-Ricci curvature tensor of the complexified Levi-Civita connection, or the second Ricci curvature of any other Hermitian connection. Quite interesting is to take $\mathcal{F}(h)=$ $s \Theta^{(1)}+(1-s) \Theta^{(2)}$ as the mixed Ricci-Chern curvature, or $\mathcal{F}(h)=B^{(2)}-2 \widehat{R}^{(2)}$ where $B^{(2)}$ is the second Ricci curvature of the Bismut connection. More generally, we can set $\mathcal{F}(h)$ to be certain suitable functions on the metric $h$. For example, if $\mathcal{F}(h)=\left(\Delta_{h} S\right) h$, the above equation will be the Hermitian Calabi flows.

The following result holds for quite general $\mathcal{F}(h)$, but here for simplicity we will only take $\mathcal{F}(h)=-\Theta^{(2)}$ as an example.

$$
\left\{\begin{array}{l}
\frac{\partial h}{\partial t}=-\Theta^{(2)}+\mu h \\
h(0)=h_{0}
\end{array}\right.
$$

where $\mu$ is a real parameter. By formula 2.38, the second Ricci-Chern curvature tensor has components

$$
\Theta_{k \bar{\ell}}^{(2)}=h^{i \bar{j}} \Theta_{i \bar{j} k \bar{\ell}}=-h^{i \bar{j}} \frac{\partial^{2} h_{k \bar{\ell}}}{\partial z^{i} \partial \bar{z}^{j}}+h^{i \bar{j}} h^{p \bar{q}} \frac{\partial h_{k \bar{q}}}{\partial z^{i}} \frac{\partial h_{p \bar{\ell}}}{\partial \bar{z}^{j}}
$$


Theorem 7.1. Let $\left(M, h_{0}\right)$ be a compact Hermitian manifold.

(1) There exists small $\varepsilon$ such that, the solution of flow $\% .11$ exists for $|t|<\varepsilon$, and it preserves the Hermitian structure;

(2) The flow 7.11 preserves the Kähler structure, i.e., if the initial metric $h_{0}$ is Kähler, then $h(t)$ are also Kähler.

Proof. (1). Let $\Delta_{c}$ be the canonical Laplacian operator on the Hermitian manifold $(M, h)$ defined by

$$
\Delta_{c}=h^{p \bar{q}} \frac{\partial^{2}}{\partial z^{p} \partial \bar{z}^{q}} .
$$

Therefore, the second Ricci-Chern curvature $-\Theta_{i \bar{j}}^{(2)}$ has leading term $\Delta_{c} h_{i \bar{j}}$ which is strictly elliptic. The local existence of the flow 7.11 follows by general theory of parabolic PDE, and the solution is an Hermitian metric on $M$.

(2). The coefficients of the tensor $\partial \omega$ are given by

$$
f_{i \bar{j} k}=\frac{\partial h_{i \bar{j}}}{\partial z^{k}}-\frac{\partial h_{k \bar{j}}}{\partial z^{i}}
$$

Under the flow 7.11, we have

$$
\left\{\begin{array}{l}
\frac{\partial f_{i \bar{j} k}}{\partial t}=\frac{\partial \Theta_{k \bar{j}}^{(2)}}{\partial z^{i}}-\frac{\partial \Theta_{i \bar{j}}^{(2)}}{\partial z^{k}}+\mu f_{i \bar{j} k} \\
f_{i \bar{j} k}(0)=0
\end{array}\right.
$$

At first, we observe that $f_{i \bar{j} k}(t) \equiv 0$ is a solution of 7.15. In fact, if $f_{i \bar{j} k}(t) \equiv 0$, then $h_{i \bar{j}}(t)$ are Kähler metrics, and so

$$
\Theta_{i \bar{j}}^{(2)}=\Theta_{i \bar{j}}^{(1)}=-\frac{\partial^{2} \log \operatorname{det}\left(h_{m \bar{n}}\right)}{\partial z^{i} \partial \bar{z}^{j}}
$$

Therefore,

$$
\frac{\partial \Theta_{k \bar{j}}^{(2)}}{\partial z^{i}}-\frac{\partial \Theta_{i \bar{j}}^{(2)}}{\partial z^{k}}=-\frac{\partial^{3} \log \operatorname{det}\left(h_{m \bar{n}}\right)}{\partial z^{i} \partial z^{k} \partial \bar{z} j}+\frac{\partial^{3} \log \operatorname{det}\left(h_{m \bar{n}}\right)}{\partial z^{i} \partial z^{k} \partial \bar{z} j}=0
$$

On the other hand,

$$
\frac{\partial \Theta_{k \bar{j}}^{(2)}}{\partial z^{i}}-\frac{\partial \Theta_{i \bar{j}}^{(2)}}{\partial z^{k}}=\Delta_{c}\left(f_{i \bar{j} k}\right)+\text { lower order terms }
$$

Hence the solution of 7.15 is unique.

Remark 7.2. Theorem 7.1 holds also for quite general $\mathcal{F}(h)$ which we will study in detail in a subsequent paper [33].

The flow 7.11 has close connections to several important geometric flows:

1. It is very similar to the Hermitian Yang-Mills flow on holomorphic vector bundles. More precisely, if the flow 7.11 has long time solution and it converges to an Hermitian metric $h_{\infty}$ such that

$$
\Theta_{i \bar{j}}^{(2)}=\mu h_{i \bar{j}}
$$

The Hermitian metric $h_{\infty}$ is Hermitian-Einstein. So, by [34], the holomorphic tangent bundle $T^{1,0} M$ is stable. As shown in Example 6.1, the Hopf manifold $\mathbb{S}^{2 n+1} \times \mathbb{S}^{1}$ is stable for any $n \geq 1$. In fact, in the definition of $\Theta_{i \bar{j}}^{(2)}$, if we take trace by using the initial metric $h_{0}$, then we get the original Hermitian-Yang-Mills flow equation. 
2. If the initial metric is Kähler, then this flow is reduced to the usual Kähler-Ricci flow $([6])$.

3. The flow 7.11 is similar to the harmonic map flow equation as shown in Theorem 7.1. It is strictly parabolic, and so the long time existence depends on certain curvature condition of the target manifold as discussed in the pioneering work of Eells-Sampson in [11]. The long time existence of this flow and other geometric properties of our new flow will be studied in our subsequent work.

Certain geometric flows and related results have been considered on Hermitian manifolds recently, we refer the reader to [43], [44], [45] and [22].

\section{Appendix: The proof of the refined Bochner formulas}

Lemma 8.1. On a compact Hermitian manifold $(M, h, \omega)$, we have

$$
[\Lambda, 2 \partial \omega]=A+B+C
$$

where

$$
\begin{gathered}
\left\{\begin{array}{l}
A=-h^{k \bar{\ell}} h_{i \bar{m}} \Gamma_{s \bar{\ell}}^{\bar{m}} d z^{s} \wedge d z^{i} I_{k} \\
\bar{A}^{*}=-h^{s \bar{t}} \Gamma_{s \bar{k}}^{\bar{i}} d \bar{z}^{k} I_{\bar{i}} I_{\bar{t}}
\end{array}\right. \\
\left\{\begin{array}{l}
B=-2 \Gamma^{\bar{\ell}} d z^{i} \wedge d \bar{z}^{j} I_{\bar{\ell}} \\
\bar{B}^{*}=2 h^{p \bar{j}} \Gamma_{\ell \bar{j}}^{\bar{s}} d z^{\ell} I_{p} I_{\bar{s}}
\end{array}\right. \\
\left\{\begin{array}{l}
C=\Lambda(2 \partial \omega)=2 \Gamma_{j \bar{\ell}}^{\bar{\ell}} d z^{j} \\
\bar{C}^{*}=2 h^{j \bar{\ell}} \Gamma_{j \bar{s}}^{\bar{s}} I_{\bar{\ell}}=-2 h^{j \bar{i}} \Gamma_{j \bar{i}}^{\bar{\ell}} I_{\bar{\ell}}
\end{array}\right.
\end{gathered}
$$

Moreover,

(1) $[\Lambda, A]=-{\sqrt{-1 B^{*}}}^{*}$

(2) $[\Lambda, B]=-\sqrt{-1}\left(2 \bar{A}^{*}+\bar{B}^{*}+\bar{C}^{*}\right)$;

(3) $[\Lambda, C]=-\sqrt{-1 C}^{*}$.

Proof. All formulas follow by direct computation.

Definition 8.2. With respect to $\nabla^{\prime}$ and $\nabla^{\prime \prime}$, we define

$$
\left\{\begin{array}{l}
D^{\prime}:=d z^{i} \wedge \nabla_{i}^{\prime} \\
D^{\prime \prime}:=d \bar{z}^{j} \wedge \nabla_{\bar{j}}^{\prime \prime}
\end{array}\right.
$$

The dual operators of $\partial, \bar{\partial}, D^{\prime}, D^{\prime \prime}$ with respect to the norm in 4.13 are denoted by $\partial^{*}, \bar{\partial}^{*}, \delta^{\prime}, \delta^{\prime \prime}$ and define

$$
\left\{\begin{array}{l}
\delta_{0}^{\prime}:=-h^{i \bar{j}} I_{i} \nabla_{\bar{j}}^{\prime \prime} \\
\delta_{0}^{\prime \prime}:=-h^{j \bar{i}} I_{\bar{i}} \nabla_{j}^{\prime}
\end{array}\right.
$$

where $I$ the contraction operator and $I_{i}=I_{\frac{\partial}{\partial z^{i}}}$ and $I_{\bar{i}}=I_{\frac{\partial}{\partial \bar{z}^{i}}}$.

Remark 8.3. It is obvious that these first order differential operators $D^{\prime}, D^{\prime \prime}, \delta_{0}^{\prime}$ and $\delta_{0}^{\prime \prime}$ are well-defined and they don't depend on the choices of holomorphic frames. If $(M, h)$ is Kähler, $D^{\prime}=\partial, D^{\prime \prime}=\bar{\partial}, \delta_{0}^{\prime}=\delta^{\prime}=\partial^{*}$ and $\delta_{0}^{\prime \prime}=\delta^{\prime \prime}=\bar{\partial}^{*}$. 
Lemma 8.4. In the local holomorphic coordinates,

$$
\partial=D^{\prime}-\frac{B}{2} \quad \text { and } \quad \bar{\partial}=D^{\prime \prime}-\frac{\bar{B}}{2}
$$

Proof. We only have to check them on functions and 1-forms.

Lemma 8.5. On a compact Hermitian manifold $(M, h)$, we have

$$
\left\{\begin{array}{l}
\delta^{\prime \prime}=\delta_{0}^{\prime \prime}-\frac{\bar{C}^{*}}{2} \\
\delta^{\prime}=\delta_{0}^{\prime}-\frac{C^{*}}{2}
\end{array}\right.
$$

For $\partial$ and $\bar{\partial}$, we have

$$
\left\{\begin{array}{l}
\partial^{*}=\delta_{0}^{\prime}-\frac{B^{*}+C^{*}}{\bar{\partial}^{2}}=\delta_{0}^{\prime \prime}-\frac{\bar{B}^{*}+\bar{C}^{*}}{2}
\end{array}\right.
$$

Proof. For any $\varphi \in \Omega^{p, q-1}(M)$ and $\psi \in \Omega^{p, q}(M)$, by stokes' theorem

$$
\begin{aligned}
0 & =\int_{M} \bar{\partial}(\varphi \wedge * \bar{\psi}) \\
& =\int_{M} \frac{\partial}{\partial \bar{z}^{j}}\left(d \bar{z}^{j} \wedge \varphi \wedge * \bar{\psi}\right) \\
& =\int_{M} \frac{\partial}{\partial \bar{z}^{j}}\left(\left\langle d \bar{z}^{j} \wedge \varphi, \psi\right\rangle \frac{\omega^{n}}{n !}\right) \\
& =\int_{M} \frac{\partial}{\partial \bar{z}^{j}}\left(\left\langle\varphi, h^{j \bar{i}} I_{\bar{i}} \psi\right\rangle \frac{\omega^{n}}{n !}\right) \\
& =\int_{M}\left(\left\langle\nabla_{\bar{j}}^{\prime \prime} \varphi, h^{j \bar{i}} I_{\bar{i}} \psi\right\rangle+\left\langle\varphi, \nabla_{j}^{\prime} h^{j \bar{i}} I_{\bar{i}} \psi\right\rangle+\left\langle\varphi, h^{j \bar{i}} I_{\bar{i}} \psi\right\rangle \frac{\partial \log \operatorname{det}\left(h_{m \bar{n}}\right)}{\partial \bar{z}^{j}}\right) \frac{\omega^{n}}{n !} \\
& =\int_{M}\left(\left\langle d \bar{z}^{j} \wedge \nabla_{\bar{j}}^{\prime \prime} \varphi, \psi\right\rangle+\left\langle\varphi, h^{j \bar{i}} \nabla_{j}^{\prime} I_{\bar{i}} \psi\right\rangle+\left\langle\varphi, \frac{\partial h^{j \bar{i}}}{\partial z^{j}} I_{\bar{i}} \psi\right\rangle+\left\langle\varphi, h^{j \bar{i}} I_{\bar{i}} \psi\right\rangle \frac{\partial \log \operatorname{det}\left(h_{m \bar{n}}\right)}{\partial \bar{z}^{j}}\right) \frac{\omega^{n}}{n !}
\end{aligned}
$$

That is

$$
\left(D^{\prime \prime} \varphi, \psi\right)=\left(d \bar{z}^{j} \wedge \nabla_{\bar{j}}^{\prime \prime} \varphi, \psi\right)=-\left(\varphi, h^{j \bar{i}} \nabla_{j}^{\prime} I_{\bar{i}} \psi\right)-\left(\varphi,\left(\frac{\partial h^{j \bar{i}}}{\partial z^{j}}+h^{j \bar{i}} \frac{\partial \log \operatorname{det}\left(h_{m \bar{n}}\right)}{\partial z^{j}}\right) I_{\bar{i}} \psi\right)
$$

Now we will compute the second and third terms on the right hand side.

$$
\frac{\partial h^{j \bar{i}}}{\partial z^{j}}+h^{j \bar{i}} \frac{\partial \log \operatorname{det}\left(h_{m \bar{n}}\right)}{\partial z^{j}}=h^{j \bar{i}} h^{s \bar{t}}\left(\frac{\partial h_{s \bar{t}}}{\partial z^{j}}-\frac{\partial h_{j \bar{t}}}{\partial z^{s}}\right)=2 h^{j \bar{i}} \Gamma_{j \bar{t}}^{\bar{t}}=-2 h^{j \bar{\ell}} \Gamma_{j \bar{\ell}}^{\bar{i}}
$$

On the other hand

$$
\begin{aligned}
-h^{j \bar{i}} \nabla_{j}^{\prime} I_{\bar{i}} & =-h^{j \bar{i}} I_{\bar{i}} \nabla_{j}^{\prime}-h^{j \bar{i}} I\left(\nabla_{j}^{\prime} \frac{\partial}{\partial \bar{z}^{i}}\right) \\
& =\delta_{0}^{\prime \prime}-h^{j \bar{i}} \Gamma_{j \bar{i}}^{\bar{\ell}} I_{\bar{\ell}}
\end{aligned}
$$

In summary, by $8.10,8.11$ and 8.12 , the adjoint operator $\delta^{\prime \prime}$ of $D^{\prime \prime}$ is

$$
\delta^{\prime \prime}=\left(\delta_{0}^{\prime \prime}-h^{j \bar{i}} \Gamma_{j \bar{i}}^{\bar{\ell}} I_{\bar{\ell}}\right)+2 h^{j \bar{i}} \Gamma_{j \bar{i}}^{\bar{\ell}} I_{\bar{\ell}}=\delta_{0}^{\prime \prime}-\frac{\bar{C}^{*}}{2}
$$


Since $\bar{\partial}=D^{\prime \prime}-\frac{\bar{B}}{2}$, we get

$$
\bar{\partial}^{*}=\delta^{\prime \prime}-\frac{\bar{B}^{*}}{2}=\delta_{0}^{\prime \prime}-\frac{\bar{B}^{*}+\bar{C}^{*}}{2}
$$

Lemma 8.6. On a compact Hermitian manifold $(M, h)$, we have

$$
\left\{\begin{array} { l } 
{ [ \Lambda , D ^ { \prime } ] = \sqrt { - 1 } ( \delta ^ { \prime \prime } + \frac { \overline { C } ^ { * } } { 2 } ) } \\
{ [ \Lambda , D ^ { \prime \prime } ] = - \sqrt { - 1 } ( \delta ^ { \prime } + \frac { C ^ { * } } { 2 } ) }
\end{array} \quad \text { and } \quad \left\{\begin{array}{l}
{\left[\delta^{\prime \prime}, L\right]=\sqrt{-1}\left(D^{\prime}+\frac{C}{2}\right)} \\
{\left[\delta^{\prime}, L\right]=-\sqrt{-1}\left(D^{\prime \prime}+\frac{\bar{C}}{2}\right)}
\end{array}\right.\right.
$$

Proof. By definition

$$
\begin{aligned}
\left(\Lambda D^{\prime}\right) \varphi & =\left(\sqrt{-1} h^{i \bar{j}} I_{i} I_{\bar{j}}\right)\left(d z^{k} \wedge \nabla_{k}^{\prime} \varphi\right) \\
& =-\sqrt{-1} h^{i \bar{j}} I_{i}\left(d z^{k} \wedge I_{\bar{j}} \nabla_{k}^{\prime} \varphi\right) \\
& =-\sqrt{-1} h^{i \bar{j}} I_{j} \nabla_{i}^{\prime} \varphi+\sqrt{-1} h^{i j} d z^{k} I_{i} I_{\bar{j}} \nabla_{k}^{\prime} \varphi \\
& =\sqrt{-1} \delta_{0}^{\prime \prime}+d z^{k} \wedge \nabla_{k}^{\prime}\left(\sqrt{-1} h^{i \bar{j}} I_{i} I_{\bar{j}} \varphi\right) \\
& =\sqrt{-1} \delta_{0}^{\prime \prime}+D^{\prime} \Lambda \varphi
\end{aligned}
$$

where we use the metric compatible condition

$$
\nabla^{\prime} \omega=0 \Longrightarrow \nabla_{k}^{\prime}(\Lambda \varphi)=\Lambda\left(\nabla_{k}^{\prime} \varphi\right)
$$

Lemma 8.7. On a compact Hermitian manifold $(M, h)$, we have

$$
\left\{\begin{array}{l}
{[\Lambda, \partial]=\sqrt{-1}\left(\bar{\partial}^{*}+\bar{\tau}^{*}\right)} \\
{[\Lambda, \bar{\partial}]=-\sqrt{-1}\left(\partial^{*}+\tau^{*}\right)}
\end{array}\right.
$$

For the dual case, it is

$$
\left\{\begin{array}{l}
{\left[\bar{\partial}^{*}, L\right]=\sqrt{-1}(\partial+\tau)} \\
{\left[\partial^{*}, L\right]=-\sqrt{-1}(\bar{\partial}+\bar{\tau})}
\end{array}\right.
$$

Proof. By Lemma 8.6, 8.4 and 8.1,

$$
\begin{aligned}
{[\Lambda, \partial] } & =\left[\Lambda, D^{\prime}\right]-\left[\Lambda, \frac{B}{2}\right] \\
& =\sqrt{-1}\left(\delta_{0}^{\prime \prime}+\frac{2 \bar{A}^{*}+\bar{B}^{*}+\bar{C}^{*}}{2}\right) \\
& =\sqrt{-1}\left(\delta^{\prime \prime}+\frac{\bar{C}^{*}}{2}+\frac{2 \bar{A}^{*}+\bar{B}^{*}+\bar{C}^{*}}{2}\right) \\
& =\sqrt{-1}\left(\bar{\partial}^{*}+\bar{\tau}^{*}\right)
\end{aligned}
$$

The other relations follow by complex conjugate and adjoint operations. 
Lemma 8.8. On an Hermitian manifold $(M, h, \omega)$,

$$
\bar{\partial}^{*} \omega=\sqrt{-1} \Lambda(\partial \omega)=\sqrt{-1} \Gamma_{\ell \bar{j}}^{\bar{j}} d z^{\ell}
$$

Proof. We have

$$
\frac{C}{2}=\Lambda(\partial \omega)=\Gamma_{j \bar{\ell}}^{\bar{\ell}} d z^{j}
$$

On the other hand, by Lemma 8.5 and $\delta_{0}^{\prime \prime} \omega=0$

$$
\begin{aligned}
\bar{\partial}^{*} \omega & =\left(\delta_{0}^{\prime \prime}-\frac{\bar{B}^{*}+\bar{C}^{*}}{2}\right) \omega=-\frac{\bar{B}^{*} \omega}{2}-\frac{\bar{C}^{*}}{2} \omega \\
& =\left(h_{\ell \bar{k}} h^{p \bar{j}} h^{i \bar{s}} \Gamma_{i \bar{j}}^{\bar{k}} d z^{\ell} I_{p} I_{\bar{s}}\right)\left(\frac{\sqrt{-1}}{2} h_{m \bar{n}} d z^{m} \wedge d \bar{z}^{n}\right)-\frac{\bar{C}^{*}}{2} \omega \\
& =-\frac{\sqrt{-1}}{2} h_{\ell \bar{k}} h^{i \bar{j}} \Gamma_{i \bar{j}}^{\bar{k}} d z^{\ell}-\frac{\bar{C}^{*}}{2} \omega \\
& =\frac{\sqrt{-1}}{2} \Gamma_{\ell \bar{j}}^{\bar{j}} d z^{\ell}-\frac{\bar{C}^{*}}{2} \omega \\
& =\sqrt{-1} \Gamma_{\ell \bar{j}}^{\bar{j}} d z^{\ell} \\
& =\sqrt{-1} \Lambda(\partial \omega)
\end{aligned}
$$

Now we assume $E$ is an Hermitian complex vector bundle or a Riemannian vector bundle over a compact Hermitian manifold $(M, h, \omega)$ and $\nabla^{E}$ is a metric connection on $E$.

Lemma 8.9. We have the following formula:

$$
\bar{\partial}_{E}^{*}(\varphi \otimes s)=\left(\bar{\partial}^{*} \varphi\right) \otimes s-h^{i \bar{j}}\left(I_{\bar{j}} \varphi\right) \wedge \nabla_{i}^{E} s
$$

for any $\varphi \in \Omega^{p, q}(M)$ and $s \in \Gamma(M, E)$.

Proof. The proof of is the same as Lemma 8.5.

Lemma 8.10. If $\tau$ is the operator of type $(1,0)$ defined by $\tau=[\Lambda, 2 \partial \omega]$ on $\Omega^{\bullet}(M, E)$, then

(1) $\left[\bar{\partial}_{E}^{*}, L\right]=\sqrt{-1}\left(\partial_{E}+\tau\right)$;

(2) $\left[\partial_{E}^{*}, L\right]=-\sqrt{-1}\left(\bar{\partial}_{E}+\bar{\tau}\right)$;

(3) $\left[\Lambda, \partial_{E}\right]=\sqrt{-1}\left(\bar{\partial}_{E}^{*}+\bar{\tau}^{*}\right)$;

(4) $\left[\Lambda, \bar{\partial}_{E}\right]=-\sqrt{-1}\left(\partial_{E}^{*}+\tau^{*}\right)$.

Proof. We only have to prove (3). For any $\varphi \in \Omega^{\bullet}(M)$ and $s \in \Gamma(M, E)$,

$$
\begin{aligned}
\left(\Lambda \partial_{E}\right)(\varphi \otimes s) & =\Lambda\left(\partial \varphi \otimes s+(-1)^{|\varphi|} \varphi \wedge \partial_{E} s\right) \\
& =(\Lambda \partial \varphi) \otimes s+(-1)^{|\varphi|} \sqrt{-1} h^{k \bar{\ell}} I_{k} I_{\bar{\ell}}\left(\varphi \wedge \partial_{E} s\right) \\
& =(\Lambda \partial \varphi) \otimes s+(-1)^{|\varphi|} \sqrt{-1} h^{k \bar{\ell}} I_{k}\left(\left(I_{\bar{\ell}}^{\varphi}\right) \wedge \partial_{E} s\right) \\
& =(\Lambda \partial \varphi) \otimes s+(-1)^{|\varphi|} \sqrt{-1} h^{k \bar{\ell}}\left(I_{k}\left(I_{\bar{\ell}}^{\varphi}\right)\right) \wedge \partial_{E} s-\sqrt{-1} h^{k \bar{\ell}} I_{\bar{\ell}}(\varphi) \wedge I_{k} \partial_{E} s \\
& =(\Lambda \partial \varphi) \otimes s+(-1)^{|\varphi|}(\Lambda \varphi) \wedge \partial_{E} s-\sqrt{-1} h^{k \bar{\ell}} I_{\bar{\ell}}(\varphi) \wedge \nabla_{k}^{E} s
\end{aligned}
$$


On the other hand

$$
\begin{aligned}
\left(\partial_{E} \Lambda\right)(\varphi \otimes s) & =\partial_{E}((\Lambda \varphi) \otimes s) \\
& =(\partial \Lambda \varphi) \otimes s+(-1)^{|\varphi|}(\Lambda \varphi) \wedge \partial_{E} s
\end{aligned}
$$

Therefore

$$
\begin{aligned}
{\left[\Lambda, \partial_{E}\right](\varphi \otimes s) } & =([\Lambda, \partial] \varphi) \otimes s-\sqrt{-1} h^{k \bar{\ell}} I_{\bar{\ell}}(\varphi) \wedge \nabla_{k}^{E} s \\
& =\sqrt{-1}\left(\left(\bar{\partial}^{*}+\bar{\tau}^{*}\right) \varphi\right) \otimes s-\sqrt{-1} h^{k \bar{\ell}} I_{\bar{\ell}}(\varphi) \wedge \nabla_{k}^{E} s \\
& =\sqrt{-1}\left(\bar{\partial}_{E}^{*}+\bar{\tau}^{*}\right)(\varphi \otimes s)
\end{aligned}
$$

where the last step follows by 8.18 .

\section{References}

[1] Alexandrov, B.; Ivanov, S. Vanishing theorems on Hermitian manifolds. Differential Geom. Appl. 14 (2001), no. 3, 251-265.

[2] Alessandrini, L.; Bassaneli, G. Metric properties of manifolds bimeromorphic to compact Kaehler manifolds, J. Diff. Geometry 37 (1993), 95-121.

[3] Alessandrini, L.; Bassanelli, G. A class of balanced manifolds. Proc. Japan Acad. Ser. A Math. Sci. 80 (2004), no. 1, 6-7.

[4] Bismut, J.-M. A local index theorem for non Kähler manifolds. Math. Ann. 284 (1989), no. 4, 681-699.

[5] Bochner, S. Vector fields and Ricci curvature, Bull. Amer. Math. Soc. 52, 776-797 (1946).

[6] Cao, H.-D. Deformation of Kähler metrics to Kähler-Einstein metrics on compact Kähler manifolds. Invent. Math. 81 (1985), no. 2, 359-372.

[7] Calabi, E.; Eckmann, B. A class of compact, complex manifolds which are not algebraic. Ann. of Math. (2) 58, (1953) 494-500.

[8] Demailly, J-P. Complex analytic and algebraic geometry. book online http://wwwfourier.ujf-grenoble.fr/ demailly/books.html.

[9] Demailly, J-P. Une preuve simple de la conjecture de Grauert-Riemenschneider. Lecture Notes in Math., 1295, 24-47, Springer, Berlin, 1987.

[10] Demailly, J.; Paun, M. Numerical characterization of the Kähler cone of a compact Kähler manifold. Ann. of Math. (2) 159 (2004), no. 3, 1247-1274.

[11] Eells, J.; Sampson, J. Harmonic mappings of Riemannian manifolds. Amer. J. Math. 86(1964) 109-160.

[12] Enrietti, N. Static SKT metrics on Lie groups. arXiv:1009.0620

[13] Fino, A.; Grantcharov, G. Properties of manifolds with skew-symmetric torsion and special holonomy. Adv. Math. 189 (2004), no. 2, 439-450.

[14] Fino, A.; Tomassini, A. Blow-ups and resolutions of strong Kähler with torsion metrics. Adv. Math. 221 (2009), no. 3, 914-935 
[15] Fino, A.; Parton, M.; Salamon, Simon Families of strong KT structures in six dimensions. Comment. Math. Helv. 79 (2004), no. 2, 317-340.

[16] Ganchev, G.; Ivanov, S. Holomorphic and Killing vector fields on compact balanced Hermitian manifolds. Internat. J. Math. 11 (2000), no. 1, 15-28.

[17] Ganchev, G.; Ivanov, S. Harmonic and holomorphic 1-forms on compact balanced Hermitian manifolds. Differential Geom. Appl. 14 (2001), no. 1, 79-93.

[18] Gauduchon, P. Le theoreme de l'excentricité nulle, C. R. Acad. Sci. Paris Ser. A, 285 (1977), 387-390.

[19] Gauduchon, P. Fibrés hermitiens à endomorphisme de Ricci non-négatif, Bull. Soc. Math. France 105 (1977), 113-140.

[20] Gauduchon, P. Hermitian connections and Dirac operators, Bol. U. M. I. ser. VII, vol. XI-B, supl. 2 (1997), 257-289.

[21] Gauduchon, P. La 1-forme de torsion d'une variété hermitienne compacte. Math. Ann. 267 (1984), no. 4, 495-518.

[22] Gill, M. Convergence of the parabolic complex Monge-Ampre equation on compact Hermitian manifolds. arXiv:1009.5756.

[23] Gray, A. Some examples of almost Hermitian manifolds. Illinois J. Math. 10 1966, 353366.

[24] Hamilton, R. Three-manifolds with positive Ricci curvature. J. Differential Geom. 17 (1982), no. 2, 255-306.

[25] Ivanov, S.; Papadopoulos, G. Vanishing theorems and string backgrounds. Classical Quantum Gravity 18 (2001), no. 6, 1089-1110.

[26] Ji, S. Currents, metrics and Moishezon manifolds. Pacific J. Math. 158 (1993), no. 2, 335-351.

[27] Ji, S.; Shiffman, B. Properties of compact complex manifolds carrying closed positive currents. J. Geom. Anal. 3 (1993), no. 1, 37-61.

[28] Jost, J.; Yau, S.-T. A nonlinear elliptic system for maps from Hermitian to Riemannian manifolds and rigidity theorems in Hermitian geometry. Acta Math. 170 (1993), no. 2, 221-254.

[29] Kobayashi, S. Differential Geometry of complex vector bundles, Iwanami Shoten, Publishers and Princeton Univ. Press. 1987.

[30] Kobayashi, S.; Nomizu, K. Foundations of differential geometry. Vol. II. Interscience Publishers John Wiley-Sons, Inc., New York-London-Sydney 1969.

[31] Kobayashy, S.; Wu, H. On holomorphic sections of certain Hermitian vector bundles, Math. Ann. 189 (1970), 1-4.

[32] Li, J.; Yau, S.-T. Hermitian-Yang-Mills connection on non-Kähler manifolds. Mathematical aspects of string theory (San Diego, Calif., 1986), 560C573, Adv. Ser. Math. Phys., 1, World Sci. Publishing, Singapore, 1987. 
[33] Li,Y.; Liu, K.; Yang, X. Hermitian geometric flow I.

[34] Liu, K.; Yang, X. Harmonic maps between compact Hermitian manifolds. Sci. China Ser. A 51 (2008), no. 12, 2149-2160.

[35] Ma, X.; Marinescu, G. Holomorphic Morse inequalities and Bergman kernels. Progress in Mathematics, 254. Birkhäuser Verlag, Basel, 2007

[36] Michelson, M.L. On the existence of special metrics in complex geometry, Acta Math. 143 (1983), 261-295.

[37] Petersen, P. Riemannian geometry. Second edition. Graduate Texts in Mathematics, 171. Springer, New York, 2006.

[38] Shiffman, B.; Sommese, A.J. Vanishing theorems on complex manifolds. Progress in Mathematics, 56. Birkhäuser 1985.

[39] Siu, Y. A vanishing theorem for semipositive line bundles over non-Kähler manifolds. J. Differential Geom. 19 (1984), no. 2, 431-452.

[40] Siu, Y-T.; Yau, S-T. Compact Kähler manifolds of positive bisectional curvature. Invent. Math. 59 (1980), no. 2, 189-204.

[41] Tosatti, V.; Weinkove, B. The complex Monge-Ampre equation on compact Hermitian manifolds. J. Amer. Math. Soc. 23 (2010), no.4, 1187-1195

[42] Tosatti, V.; Weinkove, B. Estimates for the complex Monge-Ampre equation on Hermitian and balanced manifolds. Asian J. Math. 14 (2010), no.1, 19-40.

[43] Streets, J., Tian, G., Hermitian curvature flow, arXiv: 0804.4109.

[44] Streets, J., Tian, G., A parabolic flow of pluriclosed metrics, arXiv: 0903.4418.

[45] Streets, J., Tian, G., Regularity results for pluriclosed flow, 1008.2794.

[46] Urakawa, H. Complex Laplacians on compact complex homogeneous spaces. J. Math. Soc. Japan 33 (1981), no. 4, 619-638

[47] Wu, H. Bochner technique in Differential Geometry, Math. Reports, vol.3, part 2, 1988.

[48] Yang, H-C. Complex parallelisable manifold, Proc. Amer. Math. Soc., 51(1954), 771-776.

[49] Yano, K.; Bochner, S. Curvature and Betti numbers. Annals of Mathematics Studies, No. 32. Princeton University Press, Princeton, N. J., 1953

[50] Yau, S.-T. On the Ricci curvature of a compact Kähler manifold and the complex MongeAmpre equation. I. Comm. Pure Appl. Math. 31 (1978), no. 3, 339-411.

[51] Zheng, F. Complex differential geometry. AMS/IP Studies in Advanced Mathematics, 18. American Mathematical Society, Providence, RI; International Press, Boston, MA, 2000

Department of Mathematics, University of California at Los Angeles, Los Angeles, CA, 90095-1555

E-mail Address: liu@math.ucla.edu; xkyang@math.ucla.edu 